\title{
Response of the Midlatitude Jets, and of Their Variability, to Increased Greenhouse Gases in the CMIP5 Models
}

\author{
Elizabeth A. BARnes AND LORENZO POLVANi \\ Lamont-Doherty Earth Observatory, Columbia University, Palisades, New York
}

(Manuscript received 30 July 2012, in final form 25 January 2013)

\begin{abstract}
This work documents how the midlatitude, eddy-driven jets respond to climate change using model output from phase 5 of the Coupled Model Intercomparison Project (CMIP5). The authors consider separately the North Atlantic, the North Pacific, and the Southern Hemisphere jets. The analysis is not limited to annualmean changes in the latitude and speed of the jets, but also explores how the variability of each jet changes with increased greenhouse gases.

All jets are found to migrate poleward with climate change: the Southern Hemisphere jet shifts poleward by $2^{\circ}$ of latitude between the historical period and the end of the twenty-first century in the representative concentration pathway 8.5 (RCP8.5) scenario, whereas both Northern Hemisphere jets shift by only $1^{\circ}$. In addition, the speed of the Southern Hemisphere jet is found to increase markedly (by $1.2 \mathrm{~m} \mathrm{~s}^{-1}$ between 850 and $700 \mathrm{hPa}$ ), while the speed remains nearly constant for both jets in the Northern Hemisphere.

More importantly, it is found that the patterns of jet variability are a strong function of the jet position in all three sectors of the globe, and as the jets shift poleward the patterns of variability change. Specifically, for the Southern Hemisphere and the North Atlantic jets, the variability becomes less of a north-south wobbling and more of a pulsing (i.e., variation in jet speed). In contrast, for the North Pacific jet, the variability becomes less of a pulsing and more of a north-south wobbling. These different responses can be understood in terms of Rossby wave breaking, allowing the authors to explain most of the projected jet changes within a single dynamical framework.
\end{abstract}

\section{Introduction}

The eddy-driven jets are located in the midlatitudes of both hemispheres, and their position strongly influences the synoptic conditions near the ground. The meridional position of the eddy-driven jet varies in time and describes a dominant form of internal atmospheric variability, being coupled to both stratospheric and surface dynamics (Hartmann and Lo 1998; Thompson and Wallace 2000; Baldwin and Dunkerton 1999). A range of methods have appeared in the literature to quantify this variability, with some studies using station-based sea level pressure differences (Walker 1924; Visbeck 2009), others using simple geometric statistics such as jet latitude and speed (e.g., Woollings et al. 2010) and many others using more sophisticated methods such as empirical orthogonal function (EOF) analysis to compute

Corresponding author address: Elizabeth A. Barnes, LamontDoherty Earth Observatory, Columbia University, P.O. Box 1000, Palisades, NY 10964-8000.

E-mail: eabarnes@ldeo.columbia.edu the anomaly pattern that describes the most variance (Lorenz and Hartmann 2001; Eichelberger and Hartmann 2007; Gerber et al. 2008).

In recent years, emphasis has been placed on how the mean position of the jet will respond to anthropogenic greenhouse gas emissions, and models predict a robust poleward displacement of the jets in future climates (Yin 2005; Miller et al. 2006; Swart and Fyfe 2012). Many studies have used EOF-based patterns to quantify changes in the mean state of the jet (i.e., latitude, speed, and zonal extent), by describing the response in terms of a trend in the projection of the response onto the EOF variability pattern (Miller et al. 2006; Stephenson et al. 2006; Woollings and Blackburn 2012). In fact, the discussion of the annular mode response to climate change in the Fourth Assessment Report of the Intergovernmental Panel on Climate Change [section 10.3.5.6 of Meehl et al. (2007)] focused on trends in the annular mode time series, which quantify changes in the mean jet quantities, with no discussion of the response of the jet variability patterns themselves. While quantifying the mean jet response using patterns of variability allows the 
changes in the jet to be summarized by a single number, it can give the false impression that the dominant form of jet variability will remain constant in time. The goal of this work is to show that the jet variability will also change with anthropogenic climate change.

Until recently, little emphasis has been placed on how the jet variability itself will respond to increased greenhouse gas emissions, although a few studies have investigated the changes in jet variability time scales over the next century (Gerber et al. 2010; Barnes and Hartmann 2010b). Gerber et al. (2010) found that the tropospheric variability of the southern annular mode (SAM) and northern annular mode (NAM) in a suite of chemistry climate models did not change between a present climate and global warming scenario, but their analysis focused only on the multimodel mean. Barnes and Hartmann (2011) demonstrated that jet variability in a barotropic model is a strong function of the mean jet latitude, where jets closer to the equator exhibit large meridional shifting variability while jets closer to the pole do not. Given these highly idealized experiments, it is not clear if a relationship between mean jet latitude and jet variability is present in more complex GCMs and, if so, whether the relationship is ubiquitous or confined to only certain sectors of the globe.

This work addresses three distinct questions about the midlatitude, eddy-driven jet and its variability:

- Is there a relationship between jet latitude and jet variability in state-of-the-art GCMs?

- How will the mean jet latitude and speed respond to climate change?

- How will jet variability respond to climate change?

Given that the one of the most robust results from phase 3 of the Coupled Model Intercomparison Project (CMIP3) is a poleward shift of the midlatitude jet with increasing greenhouse gases (GHG) (Meehl et al. 2007), the answers to the last two questions are potentially related. Unlike previous studies, we consider separately the North Atlantic, the North Pacific and the Southern Hemisphere jets. Furthermore, we do not limit our analysis to annualmean changes in the latitude and speed of the jets, but we explore in detail how the variability of each jet changes as greenhouse gases are increased.

\section{Data and techniques}

a. Data

\section{1) CMIP5}

The data used are the output of 79 global circulation model (GCM) integrations performed for phase 5 of the Coupled Model Intercomparison Project (CMIP5). The output available for analysis spans 26 different models from 15 different modeling groups, as detailed in Table 1. The model output analyzed in this work includes daily mean zonal wind $u$ and meridional wind $v$ at multiple pressure levels. We analyze four different forcing scenarios: preindustrial control (piControl; $25 \mathrm{yr}$ ), Historical (1980-2004; $25 \mathrm{yr}$ ), and representative concentration pathway 4.5 (RCP4.5) and 8.5 (RCP8.5) (2076-99; $24 \mathrm{yr}$ ). The RCP4.5 and RCP 8.5 scenarios correspond to futures where the global mean of the total radiative forcing due to anthropogenic emissions reaches 4.5 and $8.5 \mathrm{~W} \mathrm{~m}^{-2}$, respectively, by 2100 . A few integrations have only $20 \mathrm{yr}$ of piControl output but were nonetheless included in the analysis. Although many groups provide multiple ensemble members for each experiment, we utilize only one ensemble per model experiment here and, we limit the analysis to annual-mean results only.

\section{2) MERRA REANALYSIS}

We present observed jet statistics calculated from the Modern-Era Retrospective Analysis for Research and Applications (MERRA) reanalysis (Rienecker et al. 2011) for 1980-2004 to compare with the Historical integrations of the CMIP5 GCMs. In all cases, the jet and its variability are defined in the same way as done for the CMIP5 GCMs, and the reanalysis results are denoted by white stars in all figures.

\section{b. Definition of the jet}

The analysis focuses on three sectors of the globe: the Southern Hemisphere ( $\left.\mathrm{SH} ; 0^{\circ}-90^{\circ} \mathrm{S}\right)$, the North Atlantic $\left(0^{\circ}-90^{\circ} \mathrm{N}, 60^{\circ} \mathrm{W}-0^{\circ}\right)$, and the North Pacific $\left(0^{\circ}-90^{\circ} \mathrm{N}\right.$, $\left.135^{\circ} \mathrm{E}-125^{\circ} \mathrm{W}\right)$. These sectors are chosen because they represent three distinct regions where previous studies have focused on jet variability (Lorenz and Hartmann 2001; Hurrell et al. 2003; Eichelberger and Hartmann 2007). We construct the "eddy-driven jet" by performing a pressure-weighted average of the daily zonal winds over the 850- and 700-hPa pressure levels to obtain the nearsurface, eddy-driven component of the zonal winds in the midlatitudes denoted as $u_{850,700}$. Averaging over a deeper layer of the troposphere produces qualitatively similar results, although the location of the jet is less easily interpreted, because of the presence of subtropical westerlies aloft. Following Woollings et al. (2010), we also low-pass frequency filter the winds at each grid point using a 10-day Lanczos filter with 41 weights (Duchon 1979). This is done to remove the noise associated with individual synoptic systems, although the resulting jet latitudes are similar if this step is omitted.

\section{c. Definition of jet variability}

The term annular mode has become ubiquitous in the literature to describe a north-south wobble of the 
TABLE 1. Data used from the CMIP5 multimodel ensemble. Values denote the annual-mean jet latitude (negative latitudes are south of the equator) in the following order: Southern Hemisphere, North Atlantic, and North Pacific. Blank entries denote that the relevant data were not available for this analysis.

\begin{tabular}{|c|c|c|c|c|}
\hline Model & piControl & Historical & $\mathrm{RCP} 4.5$ & RCP8.5 \\
\hline BCC-CSM1.1 & $-47.8^{\circ}, 47.3^{\circ}, 44.1^{\circ}$ & $-48.3^{\circ}, 47.8^{\circ}, 43.5^{\circ}$ & $-48.2^{\circ}, 47.8^{\circ}, 43.8^{\circ}$ & $-49^{\circ}, 48.8^{\circ}, 44.3^{\circ}$ \\
\hline BNU-ESM & $-47.3^{\circ}, 47.2^{\circ}, 43.4^{\circ}$ & $-47.3^{\circ}, 48^{\circ}, 43.8^{\circ}$ & $-48.2^{\circ}, 48.4^{\circ}, 43.5^{\circ}$ & $-48.9^{\circ}, 49.1^{\circ}, 44^{\circ}$ \\
\hline CanESM2 & $-47.9^{\circ}, 48.6^{\circ}, 43.4^{\circ}$ & $-48.8^{\circ}, 49^{\circ}, 43.1^{\circ}$ & $-49.8^{\circ}, 49.5^{\circ}, 43.1^{\circ}$ & $-50.7^{\circ}, 50.4^{\circ}, 42.9^{\circ}$ \\
\hline CMCC-CM & - & $-48.2^{\circ}, 46.1^{\circ}, 42.4^{\circ}$ & - & $-50.5^{\circ}, 46.8^{\circ}, 43.3^{\circ}$ \\
\hline CNRM-CM5 & - & $-48.9^{\circ}, 46.1^{\circ}, 42.2^{\circ}$ & $-48.9^{\circ}, 46.2^{\circ}, 42^{\circ}$ & $-49.4^{\circ}, 46.3^{\circ}, 41.8^{\circ}$ \\
\hline CSIRO Mk3.6.0 & - & $-47.1^{\circ}, 46.5^{\circ}, 42^{\circ}$ & $-47.8^{\circ}, 47.6^{\circ}, 42.3^{\circ}$ & $-48.7^{\circ}, 47.8^{\circ}, 42.9^{\circ}$ \\
\hline FGOALS-g2 & $-43.6^{\circ}, 41.7^{\circ}, 38.7^{\circ}$ & $-44.1^{\circ}, 42.9^{\circ}, 38.9^{\circ}$ & $-44.2^{\circ}, 42.8^{\circ}, 39.8^{\circ}$ & $-45^{\circ}, 44.4^{\circ}, 41.1^{\circ}$ \\
\hline FGOALS-s2 & - & $-47.8^{\circ}, 48.3^{\circ}, 45^{\circ}$ & $-49^{\circ}, 49.2^{\circ}, 45.3^{\circ}$ & $-51.2^{\circ}, 49.9^{\circ}, 45.7^{\circ}$ \\
\hline GFDL-CM3 & - & $-49^{\circ}, 47.7^{\circ}, 40.7^{\circ}$ & - & $-51.7^{\circ}, 49.4^{\circ}, 42.8^{\circ}$ \\
\hline GFDL-ESM2G & $-49.2^{\circ}, 46.8^{\circ}, 42.7^{\circ}$ & $-50.3^{\circ}, 46.6^{\circ}, 42.5^{\circ}$ & $-51^{\circ}, 47.1^{\circ}, 43.1^{\circ}$ & $-51.5^{\circ}, 48^{\circ}, 44.3^{\circ}$ \\
\hline GFDL-ESM2M & $-48.6^{\circ}, 45.7^{\circ}, 42^{\circ}$ & $-49.5^{\circ}, 46.6^{\circ}, 41.8^{\circ}$ & $-50.4^{\circ}, 46.7^{\circ}, 42.4^{\circ}$ & $-51.2^{\circ}, 47.2^{\circ}, 42.7^{\circ}$ \\
\hline HadCM3 & - & $-49^{\circ}, 45.7^{\circ}, 40.2^{\circ}$ & - & - \\
\hline HadGEM2-CC & $-48.6^{\circ}, 49.1^{\circ}, 44^{\circ}$ & $-49^{\circ}, 48.6^{\circ}, 42.2^{\circ}$ & - & $-51.1^{\circ}, 50^{\circ}, 43^{\circ}$ \\
\hline HadGEM2-ES & $-49.2^{\circ}, 49.8^{\circ}, 43.3^{\circ}$ & - & $-50.6^{\circ}, 49.9^{\circ}, 43.3^{\circ}$ & $-51.2^{\circ}, 50.7^{\circ}, 42.7^{\circ}$ \\
\hline INM-CM4 & - & $-49.5^{\circ}, 48.8^{\circ}, 43.3^{\circ}$ & - & $-50.5^{\circ}, 49.3^{\circ}, 43.1^{\circ}$ \\
\hline IPSL-CM5A-LR & $-41.8^{\circ}, 45.9^{\circ}, 39.9^{\circ}$ & $-43.8^{\circ}, 46.9^{\circ}, 39.8^{\circ}$ & $-44.7^{\circ}, 46.9^{\circ}, 40.3^{\circ}$ & $-47.3^{\circ}, 47.8^{\circ}, 40.8^{\circ}$ \\
\hline IPSL-CM5A-MR & $-43.7^{\circ}, 46.8^{\circ}, 42.7^{\circ}$ & $-44.7^{\circ}, 46.9^{\circ}, 42.5^{\circ}$ & $-47.2^{\circ}, 47.5^{\circ}, 42.8^{\circ}$ & $-49.3^{\circ}, 47.5^{\circ}, 44^{\circ}$ \\
\hline IPSL-CM5B-LR & $-43.1^{\circ}, 44.6^{\circ}, 37.1^{\circ}$ & - & $-44.7^{\circ}, 45.4^{\circ}, 37.2^{\circ}$ & $-45.6^{\circ}, 46.9^{\circ}, 37.9^{\circ}$ \\
\hline MIROC-ESM & - & $-45.1^{\circ}, 45.3^{\circ}, 42.5^{\circ}$ & - & $-46.9^{\circ}, 46.8^{\circ}, 43.4^{\circ}$ \\
\hline MIROC-ESM-CHEM & $-44.6^{\circ}, 45.4^{\circ}, 43.4^{\circ}$ & $-45.3^{\circ}, 45.4^{\circ}, 42.5^{\circ}$ & - & $-47^{\circ}, 46.5^{\circ}, 42.7^{\circ}$ \\
\hline MIROC5 & $-47.4^{\circ}, 47.4^{\circ}, 42.8^{\circ}$ & $-47.2^{\circ}, 46.4^{\circ}, 41.8^{\circ}$ & - & $-49.8^{\circ}, 47.3^{\circ}, 43.2^{\circ}$ \\
\hline MPI-ESM-LR & $-46.6^{\circ}, 46.3^{\circ}, 43^{\circ}$ & $-47.2^{\circ}, 46.3^{\circ}, 43.1^{\circ}$ & $-47.9^{\circ}, 46.6^{\circ}, 43^{\circ}$ & $-49.4^{\circ}, 47.1^{\circ}, 43^{\circ}$ \\
\hline MPI-ESM-MR & $-46.2^{\circ}, 46.5^{\circ}, 42.9^{\circ}$ & $-47.1^{\circ}, 46.3^{\circ}, 43^{\circ}$ & $-47.6^{\circ}, 46.7^{\circ}, 42.7^{\circ}$ & $-48.7^{\circ}, 46.9^{\circ}, 43.5^{\circ}$ \\
\hline MPI-ESM-P & $-47^{\circ}, 45.5^{\circ}, 42.7^{\circ}$ & - & - & - \\
\hline MRI-CGCM3 & $-50.2^{\circ}, 47.7^{\circ}, 40.1^{\circ}$ & $-51^{\circ}, 47.2^{\circ}, 40.3^{\circ}$ & - & $-51.1^{\circ}, 47.7^{\circ}, 41.5^{\circ}$ \\
\hline NorESM1-M & - & $-51.7^{\circ}, 49.1^{\circ}, 45.9^{\circ}$ & - & $-52.8^{\circ}, 50.8^{\circ}, 47^{\circ}$ \\
\hline
\end{tabular}

midlatitude jet stream, most often defined as the leading EOF pattern of sea level pressure, geopotential height, or zonal wind. However, in many cases the jet variability is not annular (Deser 2007; Barnes and Hartmann 2010a), nor is it always true that the leading EOF must describe a north-south movement of the jet. Additionally, EOF analysis requires dual orthogonality of the patterns and time series of those patterns, and thus one may question its usefulness in describing the observed atmospheric variability, which does not operate under such tight assumptions. This work will utilize both EOF analysis and simple geometric jet statistics to quantify jet variability. It is expected that these two methods will often agree, given that they are strongly coupled even in simple stochastic models (Wittman et al. 2005). To avoid ambiguity, we will refrain from using the term annular mode and instead will refer to either the geometric jet statistics or the EOF patterns of variability.

\section{1) GeOMEtRic AnAlysis}

In the geometric view of the jet variability, we follow Wittman et al. (2005), and think of the jet as a "bump" (such as the one illustrated in Fig. 1a), characterized by three simple quantities: its width, its maximum, and the latitudinal position of the maximum. We will refer to these as the jet width, the jet speed, and the jet latitude. For clarity and consistency, the variability of these three quantities will be referred to as jet bulging, jet pulsing, and jet wobbling.

The quantities $Z_{\text {lat }}, Z_{\text {spd }}$, and $Z_{\text {wdt }}$ denote the daily time series of jet latitude, jet speed, and jet width and are defined over a given sector by zonally averaging $u_{850,700}$. An example of this is shown in Fig. 1a, where the mean zonal winds are denoted by the solid black line. The jet speed $Z_{\mathrm{spd}}$ is defined as the maximum zonal wind speed between $15^{\circ}$ and $75^{\circ}$ and the jet latitude $Z_{\text {lat }}$ is the latitude where this maximum is found (black circle). The location of maximum winds is determined by fitting a quadratic to the peak and finding the latitude of maximum wind speed at an interval of $0.01^{\circ}$. We define the jet width $Z_{\mathrm{wdt}}$ as the full width at half of the maximum jet speed (vertical dotted line). In the case where the winds never fall below half of the maximum value, the jet width is said to be undefined. In addition, we define three additional time series of the anomalous jet statistics by subtracting the seasonal cycle of each time series from itself, and we will denote variable $x$ as $\tilde{x}$ when the seasonal cycle has been removed (e.g., $\left.\tilde{Z}_{\text {lat }}\right)$. The 

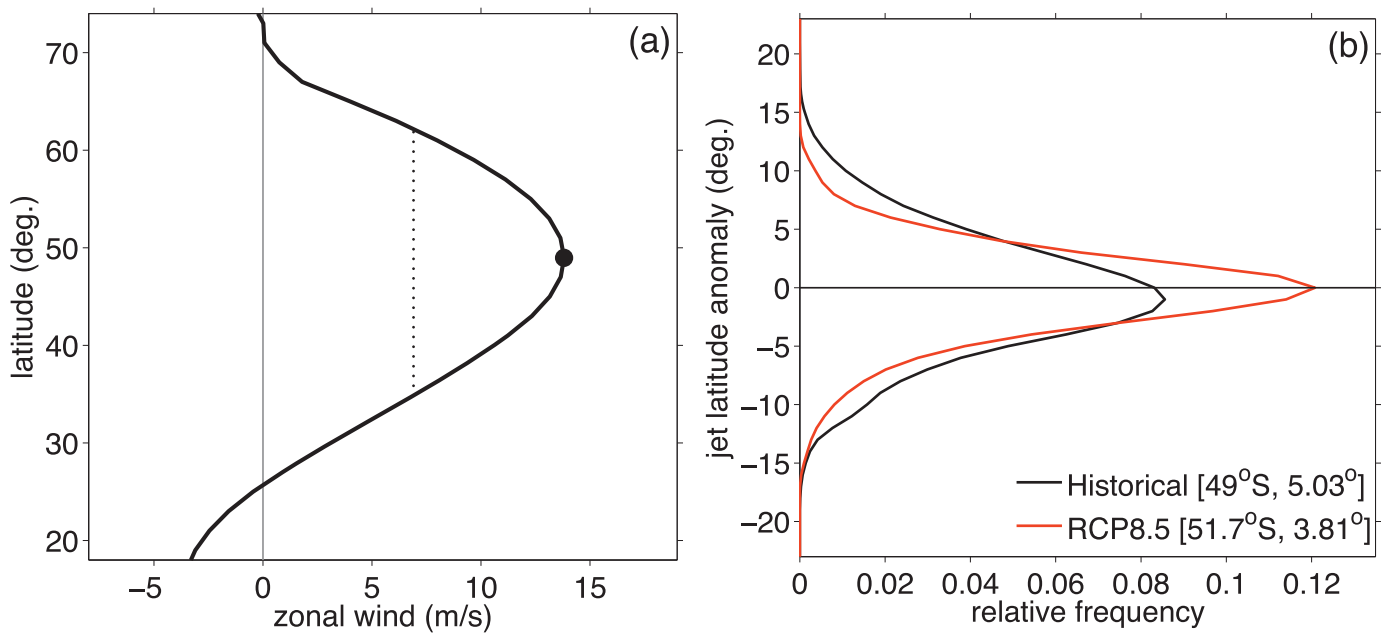

FIG. 1. (a) Schematic of a daily zonal-mean zonal wind profile. The filled circle denotes the location used to define the profile latitude and speed and the dotted line denotes the full width at half maximum. (b) Jet latitude anomaly histograms for an example model. The frequencies are normalized so that the integral under each curve equals 1 . In (b) the numbers in square brackets denote the mean jet latitude and standard deviation of the anomalous jet latitude.

seasonal cycle of each time series is defined as the mean and first four Fourier harmonics of the calendar-day mean values.

\section{2) EOF ANALYSIS}

We define variability patterns as the first and second EOF of the sector-averaged, monthly mean $\tilde{u}_{850,700}$. Daily PC time series are calculated by regressing the resulting pattern back onto the daily $\tilde{u}_{850,700}$ field. Here $Z_{\mathrm{EOF} 1}$ and $Z_{\mathrm{EOF} 2}$ denote the first and second daily PC time series, respectively, and are both normalized to unit variance. The regression patterns associated with one standard deviation of these time series will be denoted as EOF 1 and EOF 2, respectively. The EOF patterns are not sensitive to the vertical levels averaged or to the use of the monthly (instead of daily) fields.

\section{d. Rossby wave-breaking detection}

In section 6 we will discuss the response of jet variability to increased GHG forcing in the context of changes in Rossby wave-breaking frequency. We follow Barnes and Hartmann (2012) to diagnose wave breaking in the CMIP5 output. The details of the identification method, as well as comparisons with other methods, are detailed in Barnes and Hartmann (2012) and we refer the reader there for additional information. In a nutshell, the algorithm searches for overturning of the $250-\mathrm{hPa}$ absolute vorticity contours and then groups overturning contours in space and time to identify unique wave-breaking events. Using the orientation of the overturning contours, the algorithm also identifies the orientation of the overturning (cyclonic or anticyclonic).
While many previous studies have used isentropic potential vorticity to diagnose wave breaking (Wernli and Sprenger 2007; Martius et al. 2007; Strong and Magnusdottir 2008), Barnes and Hartmann (2012) demonstrate that isobaric absolute vorticity above $500 \mathrm{hPa}$ produces similar wave-breaking frequencies. This similarity is advantageous since the CMIP5 output does not include isentropic potential vorticity whereas isobaric absolute vorticity is easily computed from daily $u$ and $v$.

\section{Geometric analysis}

\section{a. Mean statistics}

The annual-mean position of the jet, defined as the temporal mean of $Z_{\text {lat }}$, is calculated for each sector, scenario, and model and is given in Table 1 for reference. The results are displayed in Fig. 2, where colored circles denote the different forcing scenarios. The black arrows connect scenarios for each model in the order of increasing GHG forcing (i.e., piControl, Historical, RCP4.5, and RCP8.5) and represent the jet shift as GHG increase. To make comparisons across sectors easier, all latitudes are plotted in degrees, where those for the Southern Hemisphere denote "degrees south" while those in the North Atlantic and North Pacific denote "degrees north".

To summarize the multimodel mean response to GHG, the left-hand side of each panel displays the multimodel mean Historical (black diagonal crosses) and RCP8.5 (red diagonal crosses) jet latitudes for models with both experiments, with an arrow denoting the average response to GHG. (Note that since all models did not 
(a) Southern Hemisphere

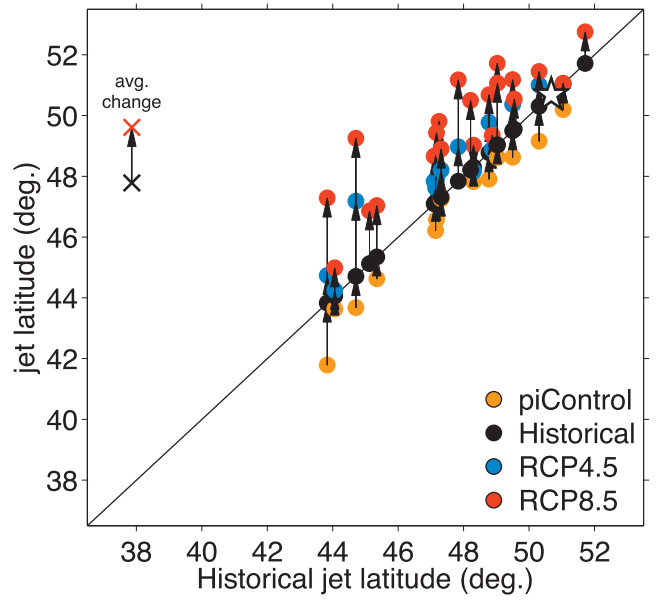

(b) North Atlantic

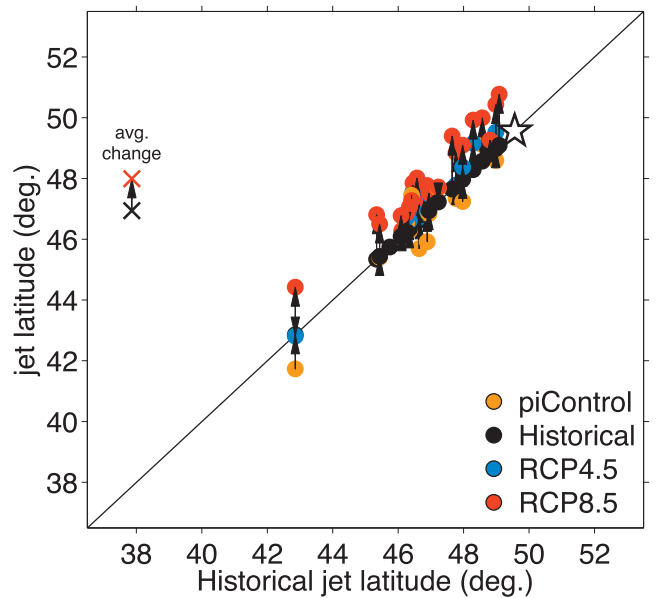

(c) North Pacific

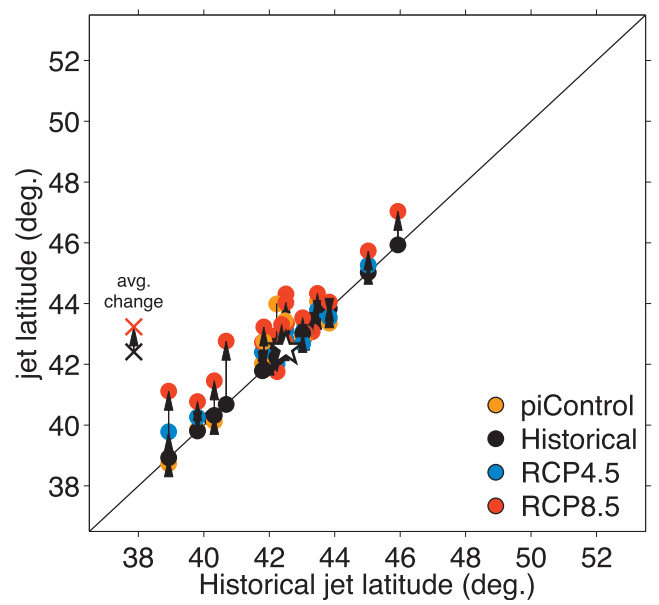

FIG. 2. Mean jet position plotted against the Historical jet latitude for (top)-(bottom) the three sectors. Arrows connect different experiments for the same model and the black and red diagonal crosses denote the multimodel mean and mean change between the Historical and RCP8.5 integrations, respectively. The solid line denotes the 1:1 line and thus the Historical jet latitude with points above denoting a poleward value and points below denoting an equatorward value. The stars denote the reanalysis. provide data for all integrations, the average change as defined here is not identical to the difference between the average RCP8.5 value and the average Historical value although they are very similar.) The key result is that between the Historical and RCP8.5 integrations, the annual-mean response in all three sectors is a poleward shift of the jet, with the jet shifting approximately $2^{\circ}$ in the Southern Hemisphere and $1^{\circ}$ poleward in the North Atlantic and North Pacific. This confirms the findings of a recent study by Swart and Fyfe (2012) where they showed a poleward shift of the Southern Hemisphere zonal-mean surface wind stress of approximately $2^{\circ}$. Similar jet shifts were documented in the Southern Hemisphere and North Atlantic using CMIP3 output (Meehl et al. 2007; Woollings and Blackburn 2012).

By using the Historical jet latitude as the abscissa, one can visualize not only the poleward jet shift, but also the spread of jet positions among the models (a range of $8^{\circ}$ in the Southern Hemisphere for the Historical period) and the deviations from the observations (white star). Interestingly, the large spread across the models is not centered near the observed jet latitude, but rather, most models place the jet equatorward of the observed position in the Southern Hemisphere and North Atlantic. This was also documented in the CMIP3 output (Barnes and Hartmann 2010c; Kidston and Gerber 2010; Woollings and Blackburn 2012).

Continuing our focus on geometric mean jet quantities, Fig. 3 depicts the annual mean of $Z_{\mathrm{spd}}$ (the wind speed) plotted against the latitude of the jet in each model integration with colors and arrows similar to those in Fig. 2. The vertical and horizontal lines denote the Historical multimodel mean values for easy comparison, and the top-right-hand corner shows the multimodel mean change (as a slope) between the Historical and RCP8.5 integrations. In the Southern Hemisphere (Fig. 3a), nearly all of the arrows point toward the topright corner of the panel, demonstrating the modelmean increase in jet speed of $1 \mathrm{~m} \mathrm{~s}^{-1}$ with a poleward jet shift of $2^{\circ}$ latitude. This increase in jet speed is not found across the globe, as shown in Figs. 3b,c where models show no systematic wind speed response in the North Atlantic and North Pacific. Woollings and Blackburn (2012) analyzed the response of the wintertime North Atlantic jet speed in the CMIP3 models and also found no robust wind speed response to increased GHG forcing.

\section{b. Variability statistics}

We now turn to the variability of the midlatitude jet and its response to increased GHG forcing. We find that the response in the Southern Hemisphere and North 
(a) Southern Hemisphere

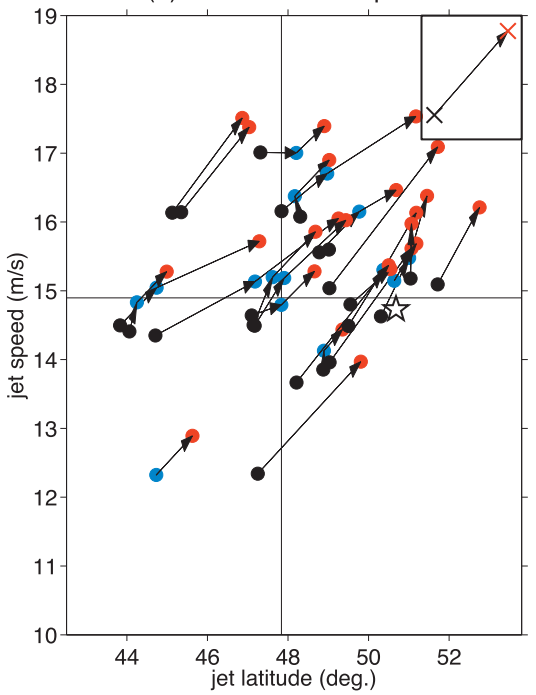

(b) North Atlantic

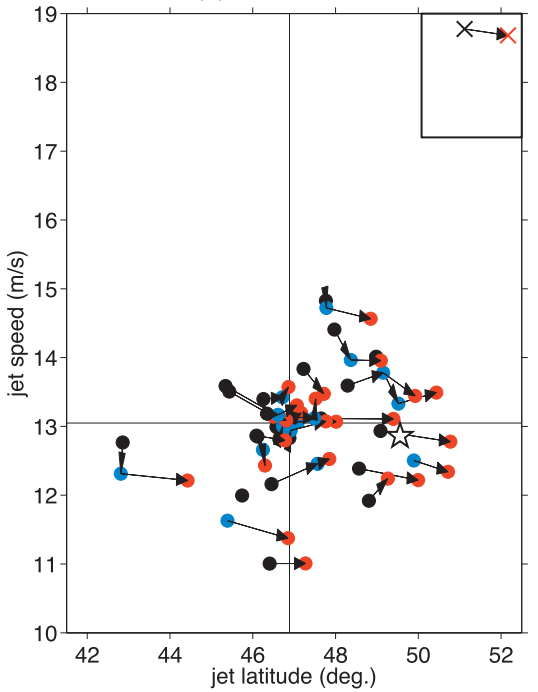

(c) North Pacific

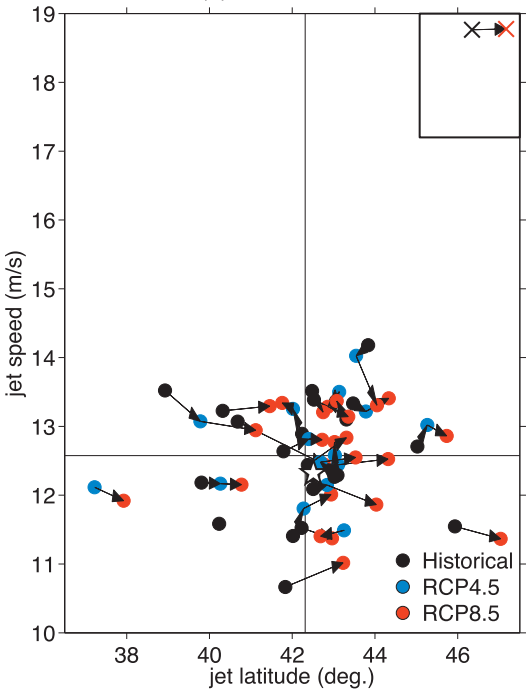

FIG. 3. Annual-mean daily jet speed vs the mean position of the jet for (left)-(right) the three sectors. Arrows connect different experiments for the same model and the black and red diagonal crosses denote the multimodel means and mean change between the Historical and RCP8.5 integrations, respectively. The solid black lines denote the model-mean values for the Historical (black) experiment and the stars denote the reanalysis. The arrows in the top-right-hand corner of each panel denote the multimodel mean change between the Historical (black) and RCP8.4 (red) scenarios.

Atlantic is similar, but that the North Pacific often displays the opposite response. For this reason, although the upcoming figures contain panels for all three sectors, we will first discuss the responses of the Southern Hemisphere and North Atlantic jet streams and will turn to the North Pacific in section 5.

Thus far, we have showed that the mean jet latitude will increase with increased GHG forcing. We now demonstrate that the variability of the jet about its mean is also predicted to change in the future, a conclusion that does not immediately follow from a change in the mean jet position. In this section we will use the simple, geometric jet quantities described in Fig. 1a to document such a change, and in the next section we will utilize a more commonly used, pattern-based EOF method to establish the robustness of the result.

As an illustration of our methods, Fig. 1b shows the smoothed histograms of daily $\tilde{Z}_{\text {lat }}$ for the Historical and RCP8.5 integrations of a single model, where the smoothing was performed with a forward and backward application of a 1-2-1 filter. The histograms have been normalized so that the area under each curve is one, with the mean jet position and standard deviation of $\tilde{Z}_{\text {lat }}$ for this particular model given in the legend. The Historical integration shows a jet latitude distribution with a wider spread (standard deviation of $5.03^{\circ}$ ) compared to the RCP8.5 curve (standard deviation of $3.81^{\circ}$ ). A smaller standard deviation of $\tilde{Z}_{\text {lat }}$ implies that the daily jet stays closer to its mean latitude and thus exhibits less meridional variability.
We calculate the standard deviation of $\tilde{Z}_{\text {lat }}$ for each integration and plot the results against the mean jet latitude for all sectors in Fig. 4. The Southern Hemisphere shows a robust decrease in the standard deviation of daily jet latitude with increased GHG forcing and poleward shift of the jet (arrows point to the bottom right). In the North Atlantic too (Fig. 3b), most models also show a decrease in the standard deviation of $\tilde{Z}_{\text {lat }}$ with increased GHG. Thus, in both the Southern Hemisphere and the North Atlantic, the jet shifts poleward and exhibits less wobbling in the future.

Furthermore, returning to the illustrative jet histograms in Fig. 1b, we find that not only does the width of the distribution of $\tilde{Z}_{\text {lat }}$ decrease between the Historical and RCP8.5 integrations, but its skewness (measure of the asymmetry about the mean) also decreases. For the Historical integration, the distribution is nearly symmetric (zero skewness), both tails approaching zero frequency $15^{\circ}$ away from the jet center. The RCP8.5 histogram, however, exhibits more frequent large negative excursions (negative skewness). The direct correlation between skewness and jet latitude is very strong, as shown for all CMIP5 integrations in both the Southern Hemisphere and North Atlantic (plotted together) in Fig. 5. In simple terms: models that place the jet closer to the equator have more positively skewed jet latitude distributions (tails toward the pole) and the skewness drops to zero and becomes negative with increasing jet latitude. Since the jet in the Southern Hemisphere tends to be farther poleward than in the North Atlantic, Fig. 5 
(a) Southern Hemisphere

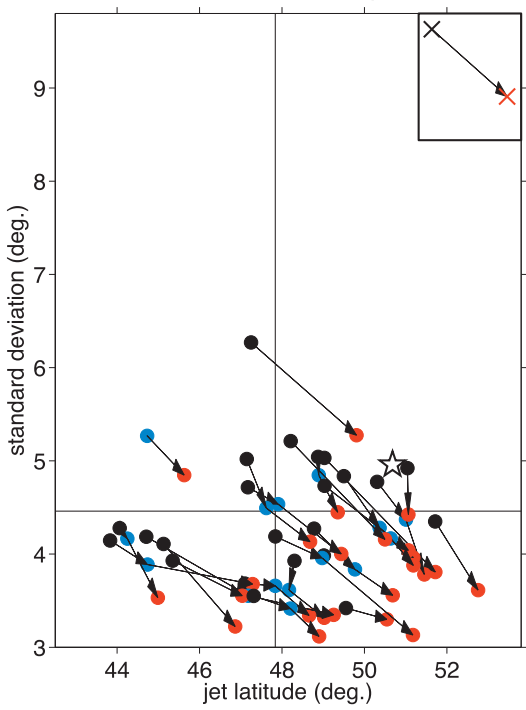

(b) North Atlantic

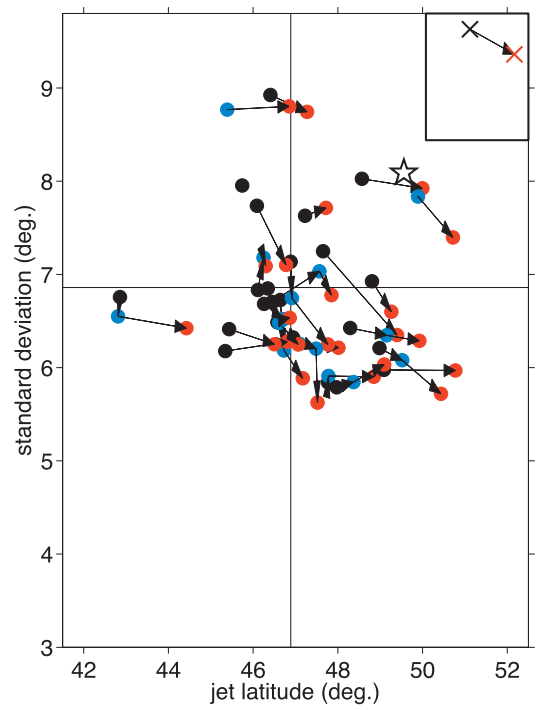

(c) North Pacific

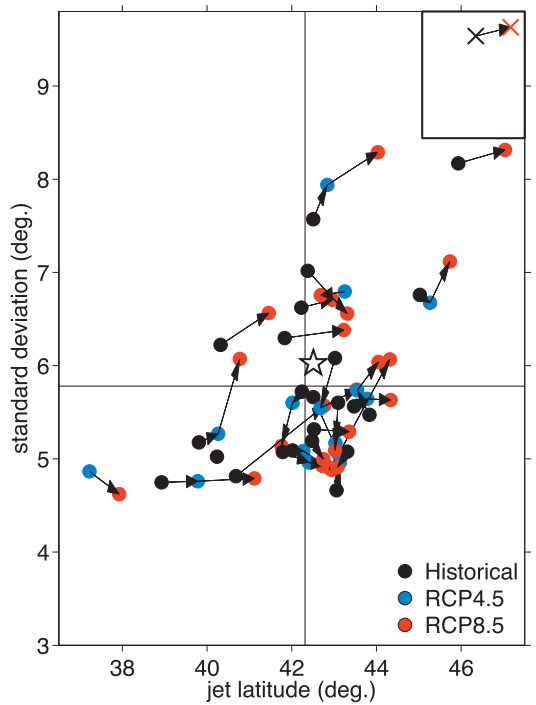

FIG. 4. As in Fig. 3, but for standard deviation of anomalous daily jet latitude vs the mean position of the jet.

demonstrates that the most negatively skewed distributions are found in the Southern Hemisphere. Interestingly, the observed skewness of the North Atlantic (circled star) falls within the range of models, while the observed skewness of the Southern Hemisphere jet latitude (star) does not.

The dependence of the standard deviation and skewness of $\tilde{Z}_{\text {lat }}$ on jet latitude implies that a poleward shift of the jet with climate change will be accompanied by a decrease in both the standard deviation and the skewness. We can visualize the jet in the future as one with less meridional variability and larger equatorward than poleward excursions.

Thus far, our analysis has focused on the variability of jet latitude, but two other metrics are required to fully document jet variability: jet speed and jet width. To quantify the importance of jet latitude, speed, and width in describing present and future jet variability, we calculate the area-weighted percent variance explained of the sector mean of $\tilde{u}_{850,700}$ by $\tilde{Z}_{\text {lat }}, \tilde{Z}_{\text {spd }}$, and $\tilde{Z}_{\text {wdt }}$ (see appendix A for details). The results are plotted against mean jet latitude in Fig. 6, where open circles denote the percent variance of the zonal winds explained by jet latitude and the filled circles denote that explained by jet speed. In both the Southern Hemisphere and North Atlantic (Figs. 6a,b), the percent variance of the zonal winds explained by jet latitude is larger than that explained by jet speed, a well-documented characteristic of the atmosphere whereby the leading pattern of jet variability describes a north-south wobble of the jet (Thompson and Wallace 2000).

The linear least squares fits are shown in Fig. 6 and give an indication of the dependence of the zonal wind variance on the mean jet latitude. The $95 \%$ confidence intervals of the slopes are given in Table 2, assuming a normally distributed random variable with mean zero and standard deviation of the slope's standard error. As the jet is found closer to the pole, the percent variance explained by jet latitude variations stays approximately constant (slope indistinguishable from zero, see Table 2) whereas that explained by jet speed variability increases in both the Southern Hemisphere and North Atlantic sectors. For the jets located at the highest latitudes in the Southern Hemisphere (Fig. 6a), the jet latitude and jet speed variability explain comparable percentages. These results agree with those presented earlier in the section, whereby the meridional variability decreases and become less important as the mean jet is located closer to the pole. Figures $6 \mathrm{a}, \mathrm{b}$ further suggest that models that place the jet at higher latitudes will have jet speed variability explain more of the variance and that the speed of the jet could explain more of the zonal wind variance in the future.

In both the Southern Hemisphere and the North Atlantic sectors, jet width explains the least amount of the variance of the zonal winds and this percentage decreases further as the jets shift poleward. The decrease in variance explained by jet width is likely linked to the small decrease in the variance explained by jet latitude since anomalous winds on the flank of the jet can result in either a widening-thinning (change in jet width) or a wobble of the maximum winds (change in jet latitude) depending on the strength of the anomaly [as demonstrated by Wittman et al. (2005), their Fig. 1d]. Thus, it is possible the two variables are documenting the same transition away from meridional variability of the jet. 


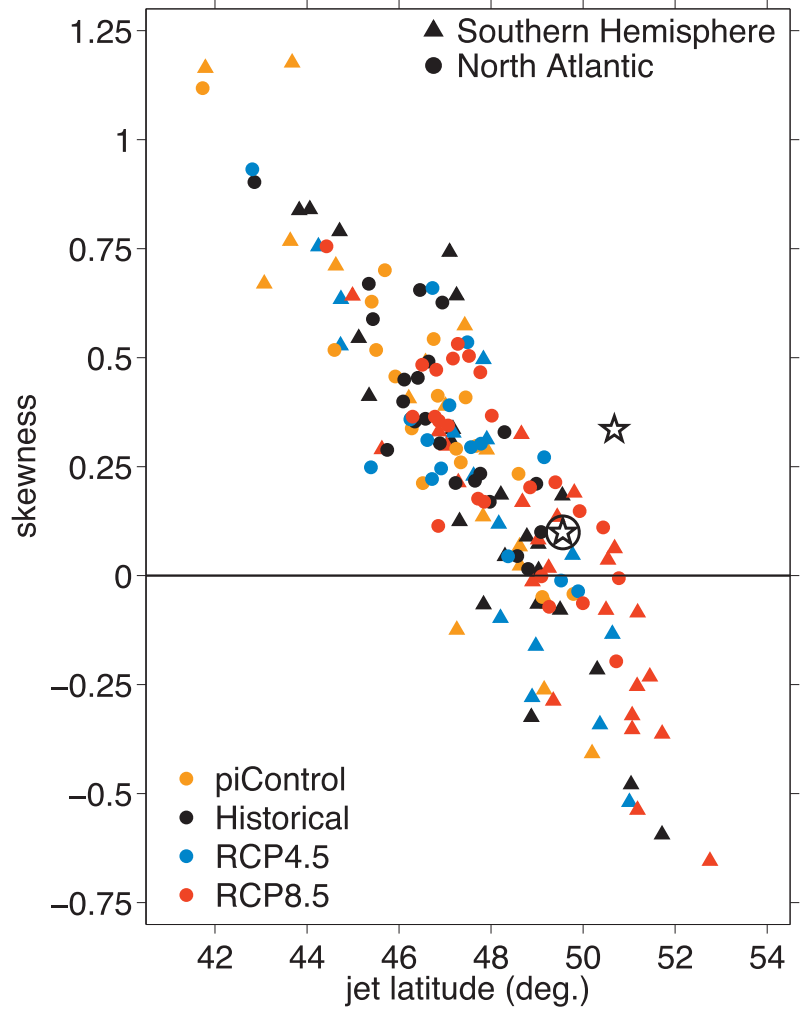

FIG. 5. Skewness of $\tilde{Z}_{\text {lat }}$ plotted against jet latitude for the Southern Hemisphere and North Atlantic sectors where the colors denote the experiment as in Fig. 2. The convention used here is that positive values represent a poleward-skewed distribution. The stars denote the reanalysis, with the plain star for the Southern Hemisphere and the circled star for the North Atlantic.

\section{EOF analysis}

Having investigated midlatitude jet variability from a geometric perspective we now turn to the more standard practice and discuss the variability of the jet in terms of the EOF patterns. These patterns of variability explain the most variance of the midlatitude zonal winds and have a long history (Thompson and Wallace 2000; Hurrell et al. 2003). In the zonal mean, the first EOF pattern usually describes a north-south wobble of the eddy-driven jet while the second EOF pattern to describes a weakening-strengthening (pulsing) of the jet (Thompson and Wallace 2000; Lorenz and Hartmann 2001, 2003).

\section{a. EOF variability and jet latitude}

\section{1) $\mathrm{EOF} 1$}

To illustrate the method, Fig. 7a shows EOF 1 patterns for the Historical and RCP8.5 integrations of the same model as in Fig. 1b. The jet in this model shifts poleward by approximately $3^{\circ}$ and so we plot the EOF 1 patterns against relative latitude, defined as degrees from the scenario-specific mean jet latitude. In addition, we normalize to give a maximum of one in order to focus on changes in the anomaly shapes.

To first order, Fig. 7a shows that the EOF 1 of the zonal winds describes the well-known north-south wobble of the jet, with positive and negative anomalies poleward and equatorward of the mean jet position. A "perfect" wobble would manifest as a zero anomaly at zero relative latitude (i.e., latitude of the mean jet). The RCP8.5 pattern in Fig. 7a exhibits a smaller wobble than the Historical pattern, with the location of the peak anomaly (filled circles) moved toward the jet center. That the EOF 1 pattern describes more of a pulse of the jet in RCP8.5 is evidenced by the larger anomaly at the jet center. Put together, the RCP8.5 variability pattern describes a smaller wobble and a larger pulsing of the jet than the Historical run.

To demonstrate the strong correlation between mean jet latitude and the jet variability pattern regardless of the forcing scenario, we compute EOF 1 for each of the 79 CMIP5 integrations, obtaining 79 profiles like those in Fig. 7a. We then group the integrations by mean jet latitude in increments of $1^{\circ}$, interpolate the profiles to a common grid and average together the EOF 1 profiles in each group. Plotting these averaged profiles against mean jet latitude yields Fig. 8, where each vertical cross section in each panel corresponds to a multimodel average EOF 1 anomaly profile. The thick line denotes the one-to-one line, and thus the latitude of the mean jet. The solid lines show the mean latitude of the maximum anomaly (multimodel mean of the dots in Fig. 7a); the thin dashed lines are parallel to the one-to-one line to aid in visualization of the slopes. The numbers along the bottom of each panel denote the number of profiles averaged in that jet latitude bin.

Looking first at the results for the Southern Hemisphere, Fig. 8a shows that EOF 1, typically called the SAM, describes a meridional wobble of the jet with the positive and negative anomalies straddling the jet center. However, regardless of the forcing scenario or model, as the mean jet is found closer to the pole, the positive anomaly moves toward the jet center (contrast thin solid and dashed lines), demonstrating a smaller jet wobble associated with EOF 1 . In the context of the North Atlantic, EOF 1 is termed the North Atlantic Oscillation (NAO) rather than an annular mode, but still describes a meridional wobble of the Atlantic jet (Deser 2007; Hurrell et al. 2003). Figure 8c shows that the NAO anomalies also move toward the jet center as the mean jet is found closer to the pole.

Using EOF analysis, we find that the pattern that describes the most variance of the zonal winds describes 
(a) Southern Hemisphere

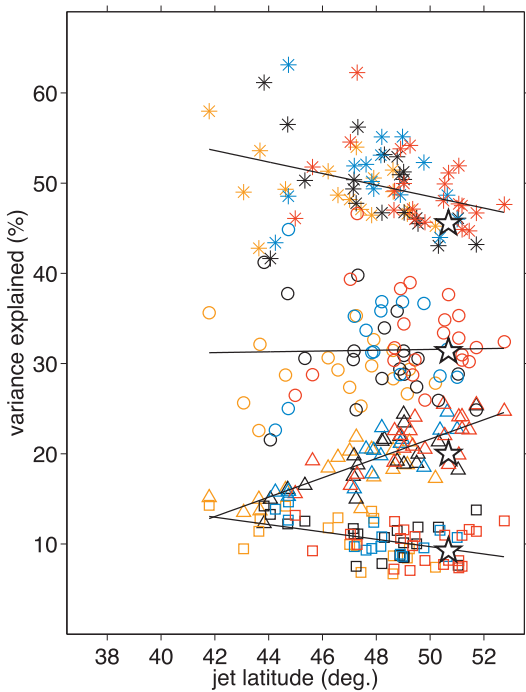

(b) North Atlantic

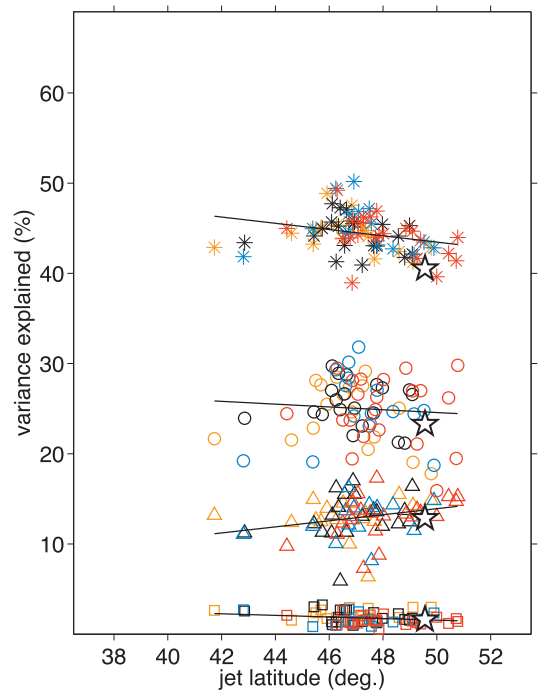

(c) North Pacific

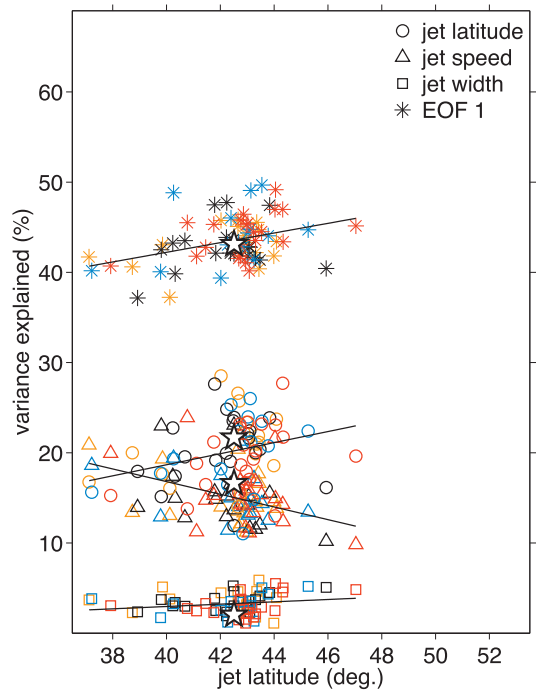

FIG. 6. Percent of the area-weighted variance of the zonal-mean zonal winds explained by $\tilde{Z}_{\text {lat }}, \tilde{Z}_{\mathrm{spd}}, \tilde{Z}_{\mathrm{wdt}}$, and $Z_{\mathrm{EOF} 1}$ vs jet latitude for (left to right) the three sectors. The straight lines denote the linear least squares fit and the colors denote the experiment as in Fig. 2.

less of a wobble and more of a pulse of the jet at higher latitudes. To compare with the results of section 3, Fig. 6 includes the percent variance of $\tilde{u}_{850,700}$ explained by $Z_{\mathrm{EOF} 1}$ (asterisks). By construction, $Z_{\mathrm{EOF} 1}$ must explain the most variance, and we see that the amount of variance explained by EOF 1 decreases with jet latitude in the Southern Hemisphere and North Atlantic as the type of variability of EOF 1 transitions from a wobble to a pulse.

\section{2) $\mathrm{EOF} 2$}

Figure $7 \mathrm{~b}$ shows sample EOF 2 patterns for the same model used in Figs. 7a and 1b. The maximum EOF 2 anomalies occur near the jet center, representing a pulsing of the jet. Since EOF 1 describes more of a pulse and less of a wobble with increased GHG, one might imagine that EOF 2 will respond in the opposite manner. Indeed, Fig. 7b shows that the EOF 2 peak anomaly moves away from the jet center for RCP8.5 (red curve), documenting a transition of EOF 2 to explaining more of a jet wobble in this model.

How EOF 2 changes with jet latitude is shown in Figs. $8 \mathrm{~b}, \mathrm{~d}$ for all CMIP5 GCMs averaged in the same way as
Figs. 8a,c, with a positive wind anomaly (warm colors) at the jet center representing variations in jet speed. As the jet is located closer to the pole, the positive anomaly moves away from the jet center, suggesting a transition of EOF 2 to describing more wobbling of the jet. We find that EOF 1 and EOF 2 together explain approximately $70 \%$ of the variance of the sector-averaged zonal winds in all sectors and integrations, and thus together describe a very significant portion of the total zonal wind variance. Putting the behavior of EOF 1 and EOF 2 together: as the mean jet is found farther poleward in both the Southern Hemisphere and North Atlantic, the EOF 1 pattern describes less of a wobble and more of a pulse of the jet, while EOF 2 does the opposite, in essence compensating for the variance no longer explained by EOF 1.

\section{b. Response of EOF 1 to climate change}

Thus far, we have not addressed how the EOF patterns themselves will respond to climate change since Fig. 8 lumps all forcing scenarios together. To this end, Fig. 9 displays the jet wobble associated with EOF 1, defined as the distance between the mean jet latitude

TABLE 2. The 95\% confidence intervals of the linear least squares regression slopes $m$ in Fig. 6 . The boldface intervals denote ranges that do not include zero. Intervals are calculated under the assumption that the random distribution of the slopes is normal with mean zero and standard deviation equal to the standard error of the slope.

\begin{tabular}{lccc}
\hline \hline & Southern Hemisphere & North Atlantic & North Pacific \\
\hline$\tilde{Z}_{\text {lat }}$ & $-0.44<m<0.53$ & $-0.62<m<0.31$ & $\mathbf{0 . 1 3}<\boldsymbol{m}<\mathbf{1 . 1 0}$ \\
$\tilde{Z}_{\text {spd }}$ & $\mathbf{0 . 8 8}<\boldsymbol{m}<\mathbf{1 . 2 7}$ & $\mathbf{0 . 0 4}<\boldsymbol{m}<\mathbf{0 . 6 3}$ & $-\mathbf{1 . 0 8}<\boldsymbol{m}<-\mathbf{0 . 3 3}$ \\
$\tilde{Z}_{\text {wdt }}$ & $-\mathbf{0 . 5 9}<\boldsymbol{m}<-\mathbf{0 . 2 2}$ & $-0.17<m<0.00$ & $-0.02<m<0.28$ \\
$Z_{\text {EOF1 }}$ & $-\mathbf{1 . 0 4}<\boldsymbol{m}<-\mathbf{0 . 2 3}$ & $-\mathbf{0 . 6 4}<\boldsymbol{m}<-\mathbf{0 . 0 6}$ & $\mathbf{0 . 2 1}<\boldsymbol{m}<\mathbf{0 . 8 6}$ \\
\hline
\end{tabular}


(a) EOF 1

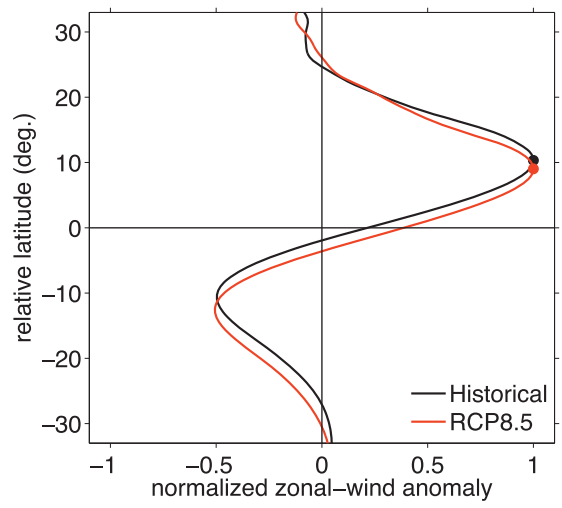

(b) EOF 2

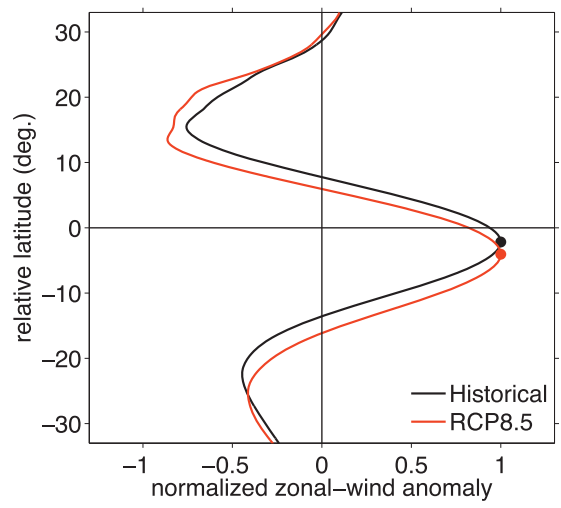

FIG. 7. Zonal wind EOF anomaly profiles associated with 1 standard deviation of (a) $Z_{\mathrm{EOF} 1}$ and (b) $Z_{\mathrm{EOF} 2}$ plotted with respect to the mean jet latitude for an example model. The curves are all normalized to equal 1 for easy comparison. Black lines denote the historical integration and red lines denote RCP8.5. The filled circles denote the location used to define the latitude of the maximum anomaly.

and the latitude of the maximum anomaly (i.e., the $y$ value of the filled circles in Fig. 7a), plotted versus the mean jet latitude.

Looking first at the Southern Hemisphere (Fig. 9a), the strong relationship between jet latitude and the EOF 1 jet wobble manifests itself as a strong linear correlation regardless of model or scenario (higher-latitude jets wobble less than lower-latitude jets). The response to climate change can be seen by following the arrows for a given model, and the multimodel mean shows a small reduction in the wobble associated with annular-mode variability although a strong relationship exists for the absolute jet wobble (regardless of scenario). This implies that the response of the EOF is determined by more than just the jet shift. A weak relationship between the absolute standard deviation and jet latitude can also be seen in Fig. 4a, in this case however, the response to climate change is more robust.

The North Atlantic is similar (Fig. 9b), with two models showing very large decreases in the magnitude of the jet wobble associated with the NAO. Again, although some models show large decreases in the EOF 1 jet wobble with increased GHG forcing, there is a hint of a relationship between the jet latitude and the absolute jet wobble, as is present in the Southern Hemisphere.

We find that the EOF 1 anomaly pattern is a function of the mean jet position and changes in the future with a poleward jet shift. Gerber et al. (2010), however, did not find a robust change in the NAM and SAM variability in a suite of chemistry climate models between a present and climate change scenario. However, they used the multimodel mean, and so even if the variability is a function of the jet position, the multimodel mean jet position shifts only a degree or two, suggesting that the signal would likely be too small to pick out among the model biases. Here, we have used the $10^{\circ}$ spread in jet latitude among the models and experiments to better see the relationship between jet latitude and jet variability. With this, however, comes the caveat that although we find a strong dependence of the EOF pattern on jet latitude, producing a large difference in jet variability from model to model, the actual expected change in jet variability is likely modest since the jet is projected to shift by no more than $4.5^{\circ}$.

\section{North Pacific jet variability}

In this section we discuss jet variability in the North Pacific sector, and how its relationship with latitude is found to be in the opposite sense to that of the Southern Hemisphere and the North Atlantic jets.

\section{a. Geometric jet analysis}

Figure $4 \mathrm{c}$ shows that the standard deviation of $\tilde{Z}_{\text {lat }}$ increases in the North Pacific between the Historical and RCP8.5 integrations, in contrast to what is found in the Southern Hemisphere and North Atlantic (Figs. 3a,b). Recall that the standard deviation of $\tilde{Z}_{\text {lat }}$ is a direct measure of the meridional variability of the jet, and thus an increase in the North Pacific implies that the jet will wobble more latitudinally in the future. Given this result, we might expect the amount of variance of $\tilde{u}_{850,700}$ explained by jet latitude to increase, and that explained by jet speed to decrease with latitude in the North Pacific. Indeed, Fig. $6 \mathrm{c}$ shows that the variance explained by jet latitude (open circles) increases and the variance 
(a) Southern Hemisphere

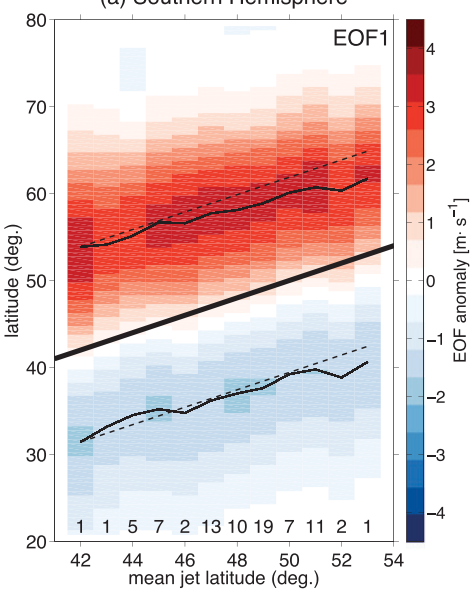

(c) North Atlantic

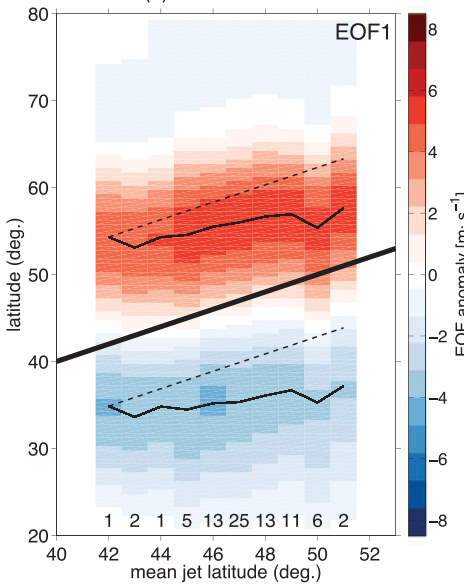

(e) North Pacific

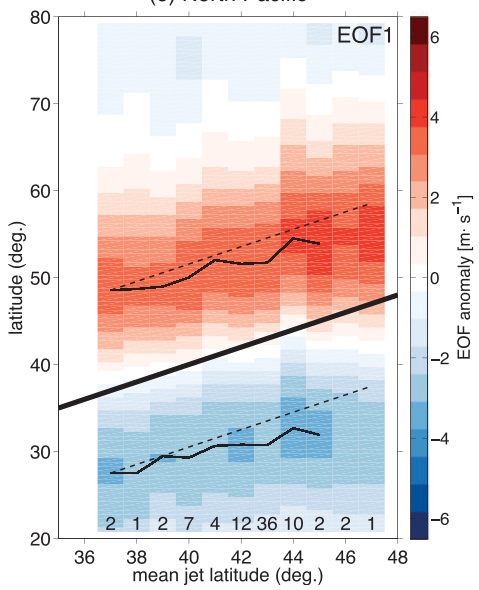

(b) Southern Hemisphere

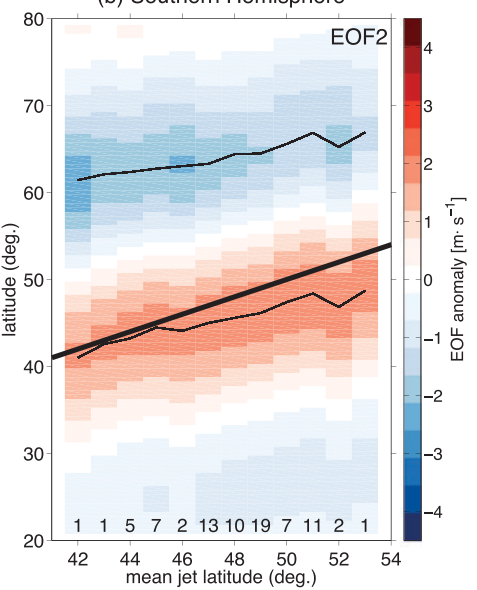

(d) North Atlantic

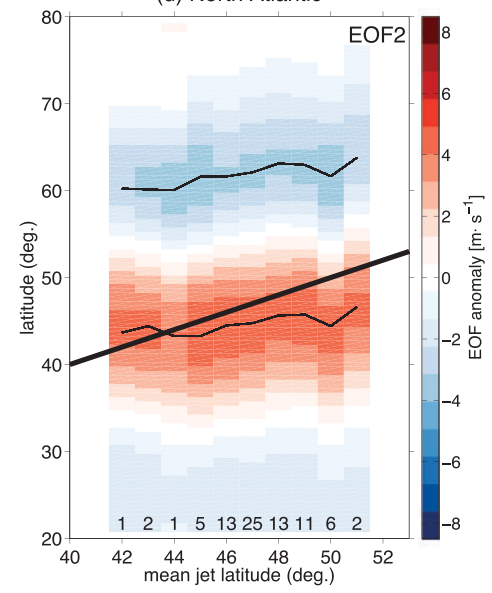

(f) North Pacific

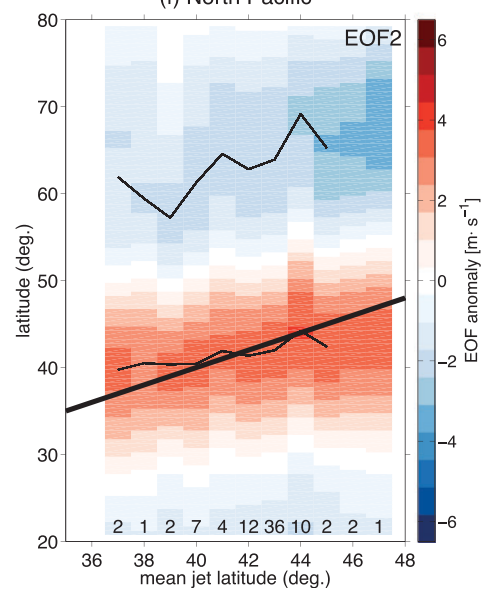

FIG. 8. Anomalous zonal winds for (a),(c),(e) EOF 1 and (b),(d),(f) EOF 2 vs mean jet latitude, where model results have been grouped into $1^{\circ}$ jet latitude bins and the profiles averaged together: (a),(b) Southern Hemisphere; (c),(d) North Atlantic; and (e),(f) North Pacific. The number of profiles in each jet latitude bin is denoted along the $x$ axis. The thin solid lines show the average anomaly-peak latitudes for each jet latitude bin, calculated by first finding the anomaly peaks for each integration and then averaging the peak latitudes. The thick line denotes the 1:1 line and thus the position of the mean jet and the thin dashed lines in (a),(c),(e) are near-parallel to the 1:1 line to aid in visualization of the equatorward slopes of the EOF anomalies. 
(a) Southern Hemisphere

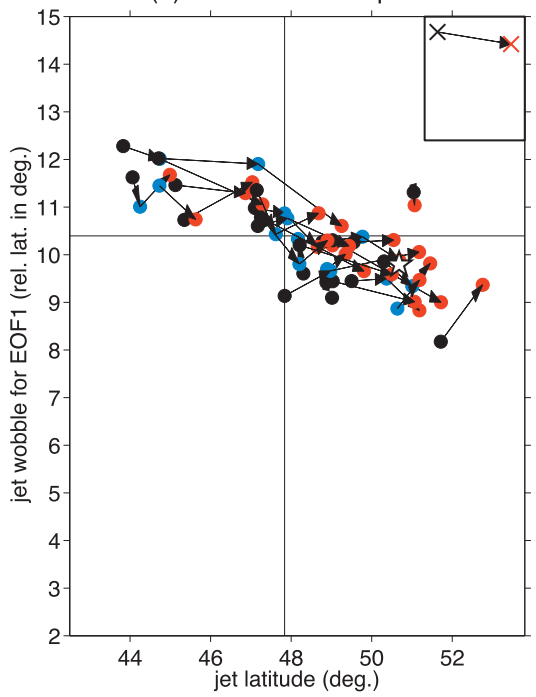

(b) North Atlantic

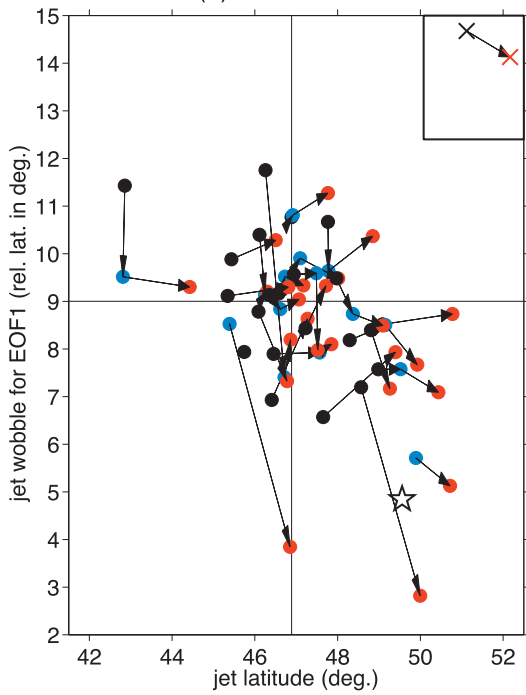

(c) North Pacific

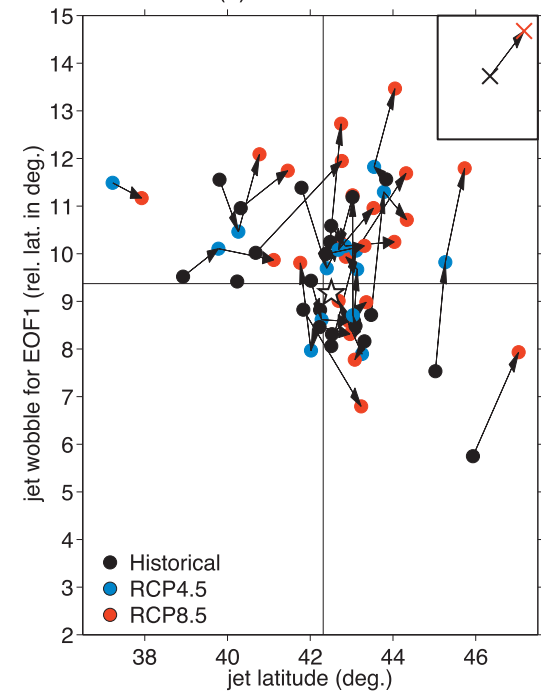

FIG. 9. As in Fig. 3, but for the meridional displacement (wobble) from the mean jet latitude of the anomalous wind profiles associated with 1 standard deviation of $Z_{\mathrm{EOF} 1}$.

explained by jet speed (closed circles) decreases for jets at higher latitudes, with both slopes being statistically different from zero (see Table 2).

\section{b. EOF analysis}

The first and second EOFs of $\tilde{u}_{850,700}$ in the North Pacific sector are plotted against the mean jet latitude in Figs. 8e,f. Similar to the other two sectors, on the whole EOF 1 describes mostly meridional wobbles while EOF 2 describes a pulsing of the jet. However, in the North Pacific, the EOF 1 and EOF 2 anomalies (relative to the mean jet latitude) vary relatively little with jet latitude (approximately constant slopes of thin solid lines). We have omitted drawing the peak anomaly latitude line for the two most poleward jet integrations in this sector because these two points are associated with the model outlier in Fig. 2c, and they visually skew the interpretation of the other 70 model integrations. However, the EOF patterns for these outliers are still presented in the colored shading for reference.

Figure $6 \mathrm{c}$ shows that the amount of variance explained by EOF 1 in the North Pacific increases with jet latitude, opposite to the other two basins that show decreases. So, although there is no strong dependence of the EOF 1 pattern itself on latitude in the North Pacific, the ability of the pattern to describe the zonal wind variance increases as the mean jets move poleward.

Although there is no strong dependence of the EOF 1 anomaly pattern on the jet latitude in the North Pacific, there is a robust response to climate change. Figure 9c shows that the magnitude of the wobble associated with
EOF 1 in the North Pacific increases between the Historical and RCP8.5 scenarios, a response that is in the opposite direction of the Southern Hemisphere and North Atlantic responses. At first glance, the results from Figs. $8 \mathrm{e}$ and $9 \mathrm{c}$ appear contradictory. However, the two figures are not necessarily at odds since Fig. 8e shows that there is no strong dependence of variability on jet latitude when all models and integrations are grouped together, while Fig. 9c demonstrates that when each model and its inherent biases are considered separately, there is a systematic increase in the magnitude of the EOF 1 wobble.

\section{Mechanism for response}

In summary, the above analysis of the CMIP5 models shows that the response of the midlatitude jet to increased GHG forcing in the Southern Hemisphere and North Atlantic is a poleward shift of the jet and a reduction in the meridional variability of the jet. In the North Pacific, the jet also shifts poleward with climate change, however, the meridional variability of the jet increases with jet latitude. In this section we propose a single mechanism to explain these different responses.

\section{a. Previous work}

Barnes and Hartmann (2011) stirred a barotropic model on the sphere to create an eddy-driven jet that exhibits annular-mode-like variability, as first demonstrated by Vallis et al. (2004). The persistence of the wobbling jet is maintained through a positive eddy-mean 
flow feedback, whereby the speed of the background winds influences where the waves propagate and break (i.e., at the critical latitudes where the phase speed of the wave is equal to the background zonal wind) and this breaking creates momentum fluxes that feed back onto the jet and reinforce the westerlies (Hartmann 2007). This positive feedback is what allows the jet to wobble and persist at a new location, even in a barotropic model.

Barnes and Hartmann (2011) showed that as the eddydriven jet is found closer to the pole in the barotropic model, the meridional movement of the jet is reduced and the jet speed explains more of the variance of the zonal winds while the jet latitude explains less, just as we have documented above for the CMIP5 models in the Southern Hemisphere and North Atlantic. Garfinkel et al. (2013) confirmed this relationship between jet variability and jet latitude in an idealized modeling study, where they varied the equilibrium temperature profile to shift the eddy-driven jet and found that jet speed explained the most variance of the zonal-mean zonal wind for high- and low-latitude jets, while jet wobbling explained the most variance for jets near $40^{\circ}$ latitude (see their Fig. 12).

Barnes and Hartmann (2011) suggest that the background vorticity gradient on which tropospheric Rossby waves propagate decreases drastically near the pole due to geometric effects, and so the waves are unable to propagate and break on the poleward flank of the jet. This lack of polar wave breaking severs the feedback loop between the waves and the jet, and thus renders the jet unable to "wobble and stick" as it does nearer the equator. A subsequent study by Barnes and Hartmann (2012) examined the number of wave-breaking events in this barotropic model and confirmed that wave breaking on the poleward flank of the jet vanishes for high-latitude jets. Thus, jets at high latitudes experience weaker meridional variability because of their weaker eddy feedbacks from the lack of wave breaking near the pole.

A region of particular interest for studying eddymean flow feedbacks is the North Pacific, where a strong subtropical jet resides during the winter season and is merged with the eddy-driven jet. Eichelberger and Hartmann (2007) demonstrated that the presence of strong subtropical winds during wintertime causes the leading mode of jet variability in the North Pacific to be a pulsing of the jet, rather than a meridional wobble. To test this mechanism in a barotropic model, Barnes and Hartmann (2011) present an additional experiment, where they introduce a band of strong westerlies in the subtropics of the stirred barotropic model to mimic a strong subtropical jet. Intriguingly, as they move the eddy stirring from the midlatitudes into the subtropics, the magnitude of the eddy-driven jet wobbling decreases, and jet pulsing becomes the dominant pattern of variability. They argue that for a low-latitude jet, the subtropical winds set the equatorward critical line, and as such, variations in the eddy-driven winds do not greatly influence the latitude of wave breaking. This once again reduces the positive feedback between the eddies and the meridional movement of the eddy-driven winds.

\section{b. Wave breaking in the CMIP5 models}

So far, the CMIP5 results confirm those of the barotropic and idealized modeling studies (Garfinkel et al. 2013; Barnes and Hartmann 2011), namely, that the variability of the eddy-driven jet is a strong function of the mean jet latitude itself. To demonstrate that the wave-breaking mechanism of Barnes and Hartmann (2011) applies to the CMIP5 models, we diagnose changes in wave behavior in the CMIP5 models using the wavebreaking detection algorithm of Barnes and Hartmann (2012) (see brief discussion in section 2). We note that Barnes and Hartmann (2012) published wave-breaking frequencies for CMIP3 output and obtained similar frequencies to those from CMIP5, but we present the CMIP5 results for completeness.

The zonally integrated wave-breaking frequencies for the Southern Hemisphere and North Atlantic are shown in Figs. 10a,b,d,e. As in Fig. 8, the binned histograms of wave-breaking frequency from each integration have been grouped together by mean jet location and averaged together. Figures 10a,d show anticyclonic wavebreaking frequencies, which preferentially appear on the anticyclonic (equatorward) flank of the jet (solid line). As one might expect, the midlatitude wavebreaking frequencies shift with the jet in a nearly oneto-one fashion, which is to be expected if the feedback between the eddies and the jet remains intact.

Figures 10b,e display the cyclonic wave-breaking frequencies, which occur predominantly on the cyclonic (poleward) flank of the jet. It is clear that the wave breaking on the poleward jet flank does not shift with the jet, but rather, stays fixed around $60^{\circ}$ latitude and just fizzles out as the jet latitude moves poleward. This decrease in high-latitude wave breaking is clear in Figs. $10 \mathrm{c}, \mathrm{f}$, which show the number of cyclonic wave-breaking events poleward of $20^{\circ}$ latitude as a function of jet position and forcing scenario. Most models exhibit a decrease in cyclonic wave breaking with increased GHG forcing in the Southern Hemisphere and North Atlantic, with a multimodel mean decrease of approximately $10 \%$ in both sectors. For reference, we have included similar plots for the eddy-momentum fluxes in appendix B for comparison with the Rossby wave-breaking analysis. 
(a) Southern Hemisphere

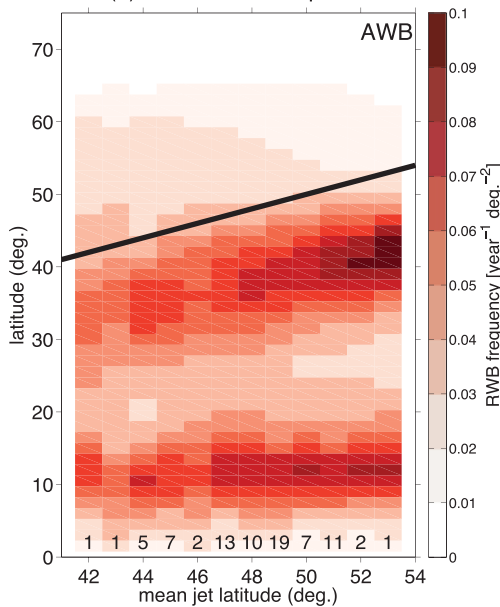

(d) North Atlantic

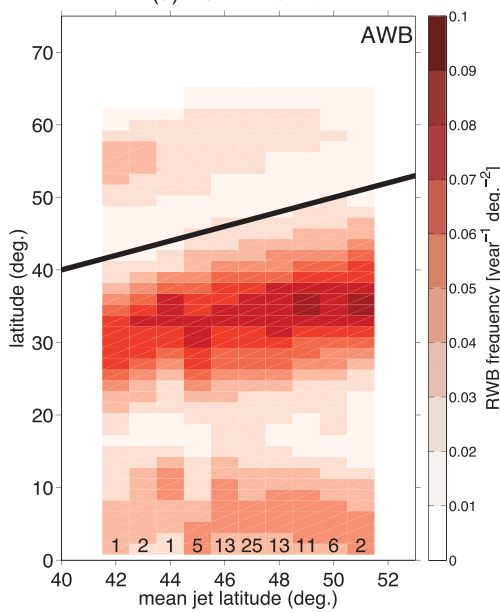

(g) North Pacific

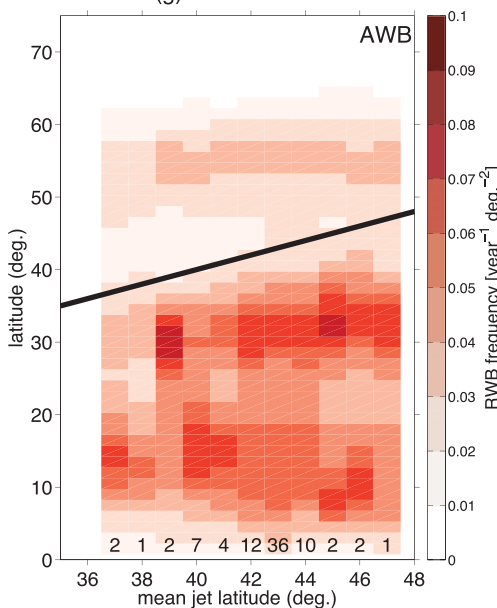

(b) Southern Hemisphere

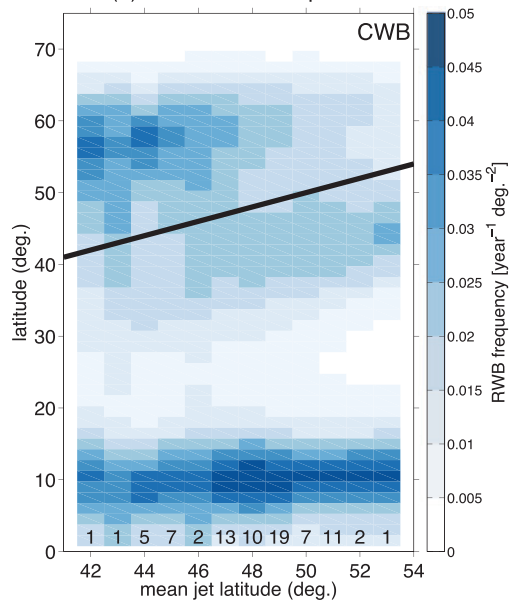

(e) North Atlantic

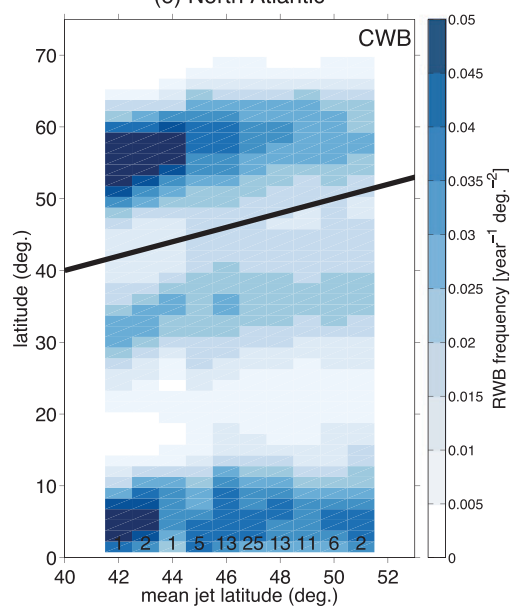

(h) North Pacific

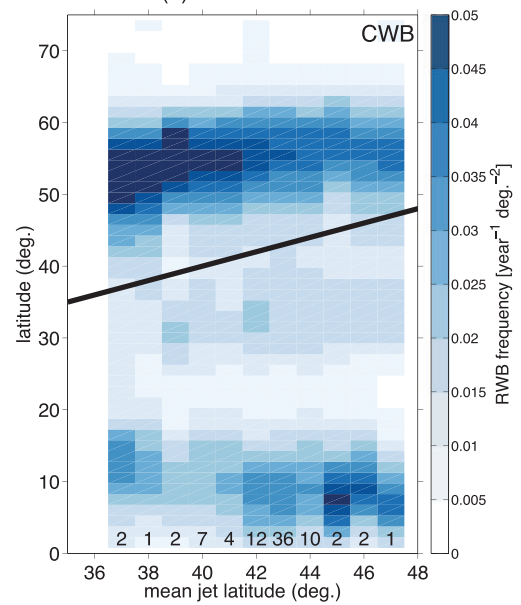

(c) Southern Hemisphere

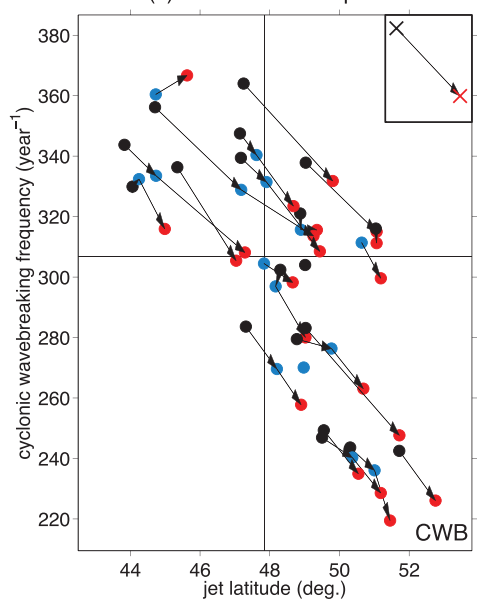

(f) North Atlantic

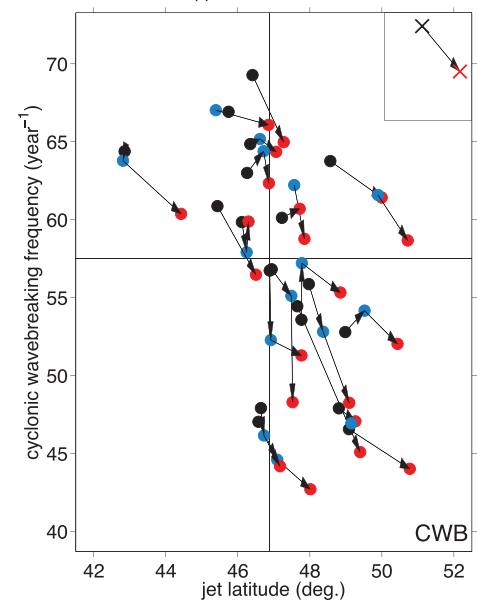

(i) North Pacific

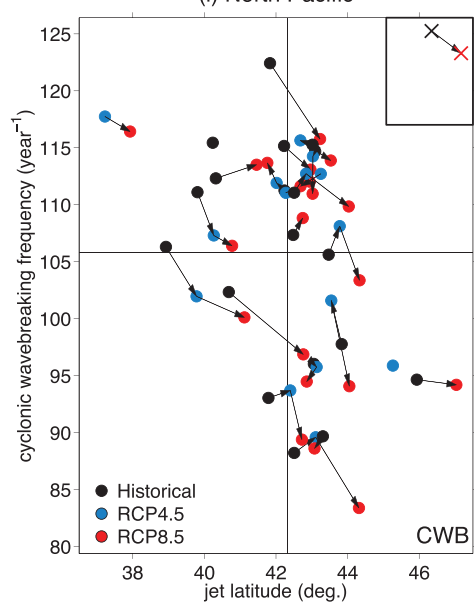

FIG. 10. Zonally integrated wave-breaking frequency profiles for (a),(d),(g) anticyclonic wave breaking (AWB) and (b),(e),(h) cyclonic wave breaking (CWB) vs mean jet latitude, where model results have been grouped into $1^{\circ}$ jet latitude bins and the profiles averaged together: (a)-(c) Southern Hemisphere; (d)-(f) North Atlantic; and (g)-(i) North Pacific. The number of profiles in each jet latitude bin is denoted along the $x$ axis. The thick line denotes the 1:1 line and thus the position of the mean jet. (c),(f),(i) Cyclonic wave-breaking frequency poleward of $20^{\circ}$ latitude with arrows, lines and colors are as in Fig. 3. 


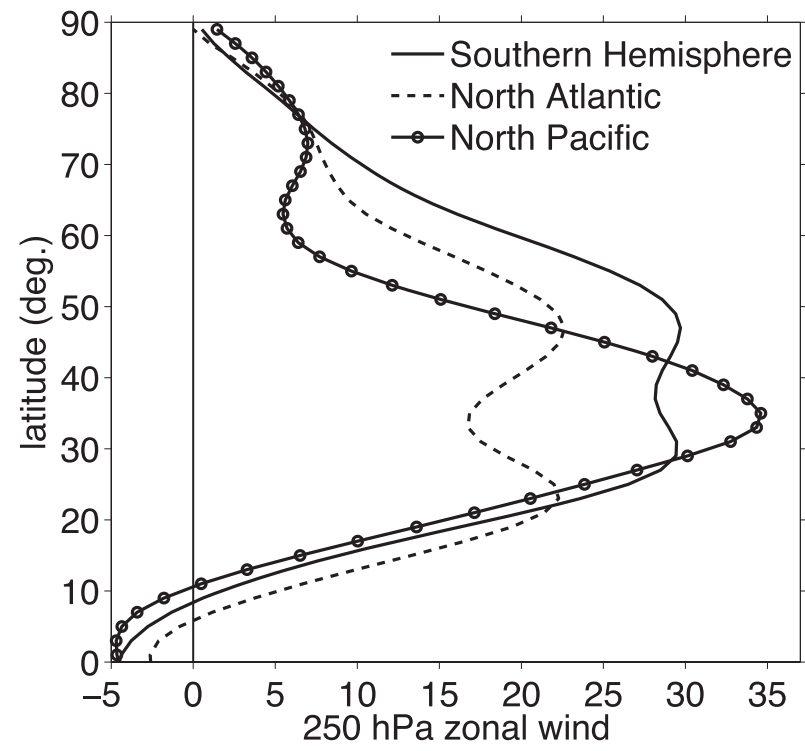

FIG. 11. Annual-mean 250-hPa sector-averaged zonal wind for the Historical integration of an example model.

In both the Southern Hemisphere and North Atlantic, the cyclonic wave breaking on the poleward flank of the jet decreases as the mean jet is located at higher latitudes. Along with these changes, the meridional variability of the jet decreases, and the jet speed explains more and more of the zonal wind variability. We suggest the mechanism of Barnes and Hartmann (2011): the positive eddy-mean flow feedback is reduced when the absolute vorticity gradient is too weak as a result of the spherical geometry (the gradient of planetary vorticity is very small near the pole) and thus inhibits synoptic wave breaking on the poleward flank of the jet. A decreased positive feedback implies a reduction in the meridional excursions of the jet maintained by this feedback, and indeed, results from the earlier sections support this conclusion.

We now come to the North Pacific, where the response of the midlatitude jet variability is nearly opposite that of the Southern Hemisphere and North Atlantic. The reason for this is the presence of a strong subtropical jet in the North Pacific that inhibits the eddy-mean flow feedback. Figure 11 displays the sectoraveraged $250-\mathrm{hPa}$ zonal winds for the same model in Figs. $1 \mathrm{~b}$ and 7 . The annual-mean North Pacific exhibits a single, strong jet at $250 \mathrm{hPa}$, while there is a clear separation between the subtropical and midlatitude jets in the Southern Hemisphere and North Atlantic, with the poleward maxima associated with the eddy-driven jet. Recall that the eddy-driven jet in the North Pacific is significantly equatorward of the midlatitude jets in the Southern Hemisphere and North Atlantic (see Fig. 2), and we see that manifested in the $250-\mathrm{hPa}$ winds in accordance with the barotropic structure of the eddydriven winds.

The wave-breaking frequencies in the North Pacific are displayed in Figs. 10g-i. Given the placement of the North Pacific jet at lower latitudes, the frequency of cyclonic wave breaking on the poleward flank of that jet is larger than in the other two sectors and decreases only slightly (less than $3 \%$ ) with climate change. However, anticyclonic wave breaking on the equatorward flank of the jet (Fig. 10g) is smeared over the subtropics in comparison to the other two sectors (Figs. 10a,d) and does not shift with the jet. This is because the subtropical and eddy-driven jets overlap in the North Pacific, and thus there is no clear separation between the waves that break on the equatorward flank of the eddy-driven jet and those that break on the equatorward flank of the subtropical jet, as is the case elsewhere. This further supports our claim that the strong subtropical winds dominate the propagation and wave-breaking characteristics of the eddies in this sector.

In summary, the mechanism proposed here places the eddy-driven jet and its variability into one of the following three regimes:

1) A high-latitude eddy-driven jet where the meridional variability is suppressed by the presence of a weak background vorticity gradient near the pole.

2) A low-latitude eddy-driven jet where the meridional variability is suppressed by the presence of strong subtropical winds.

3) An unconstrained, meridionally wobbling eddy-driven jet found somewhere in between.

The response of the eddy-driven jet variability to climate change depends strongly on where the observed jet lies within these three regimes. Higher-latitude jets in the Southern Hemisphere and North Atlantic fall into the third category and transition to the first category as the jets shift poleward with climate change, while the lower latitude North Pacific jets fall into the second category and transition to the third category.

\section{Seasonality}

Our results have highlighted the annual-mean response of the eddy-driven jet and its variability in the three sectors. However, jet variability and the strength of the subtropical winds are also functions of season. For instance, Woollings and Blackburn (2012) have documented that the poleward shift of the eddy-driven jet in the North Atlantic is smaller in winter than in summer in the CMIP3 models. The seasonality of the jet shift among the CMIP5 models is shown in Fig. 12. The bars 


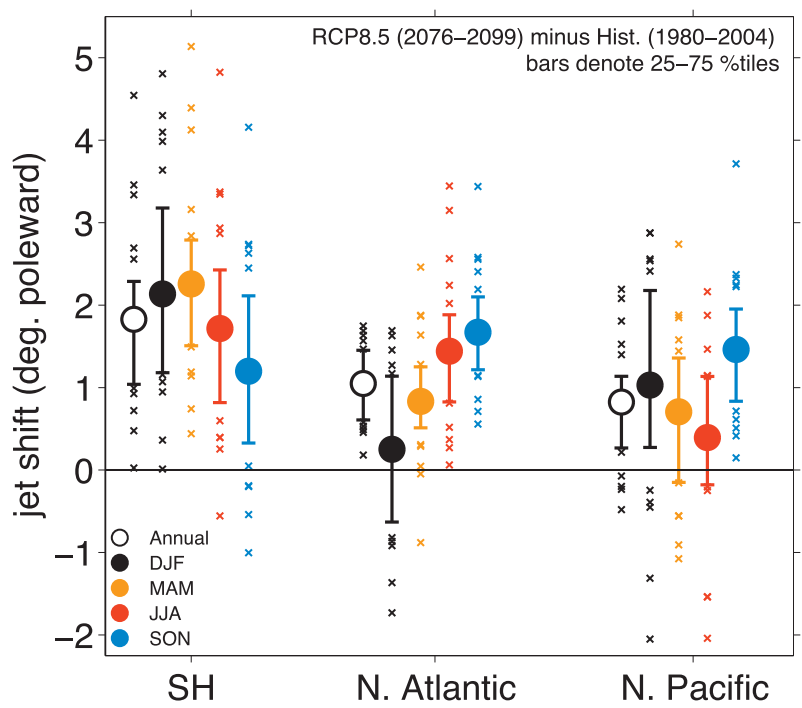

FIG. 12. Seasonal-mean jet shift (degrees poleward) between the Historical and RCP8.5 experiments for the three sectors. The bars denote the 25th-75th percentile range of the multimodel spread ( 22 models total) and the diagonal crosses denote the models outside of this range.

denote the 25 th -75 th percentile range, while the crosses denote those models that lie outside of that range. Seasonal differences are readily apparent, with the maximum jet shift occurring in autumn in all three sectors [March-May (MAM) for the Southern Hemisphere and September-November (SON) for the Northern Hemisphere], and most notably, the North Atlantic jet showing no clear shift in DJF by the end of the twenty-first century. Consistent with the jet variability being a function of the mean jet latitude, we do not find a consistent response in jet variability in the wintertime North Atlantic (not shown). Thus, it is clear that the annual-mean results from this study mask rich seasonality among the model responses, and future work should address how the story differs among the seasons in each sector. However, this additional work is beyond the scope of this paper.

We will, nonetheless, address one aspect of the seasonality of the jet variability response, namely that of the North Pacific. We noted above that the North Pacific jet response differs from the North Atlantic and Southern Hemisphere jet responses due to the presence of a strong subtropical jet in the annual mean. However, the subtropical jet has a seasonal cycle, maximizing in the winter months [December-February (DJF)]. Thus, one might expect the North Pacific summertime [JuneAugust (JJA)] jet to behave more like the Southern Hemisphere and North Atlantic jets when the subtropical jet is weak. Figure 13 shows the percent variance explained of $u_{850,700}$ by $\tilde{Z}_{\text {lat }}, \tilde{Z}_{\mathrm{spd}}, \tilde{Z}_{\mathrm{wdt}}$, and $\tilde{Z}_{\mathrm{EOF} 1}$ in the North Pacific for DJF and JJA. The jet is farther (a) North Pacific, DJF

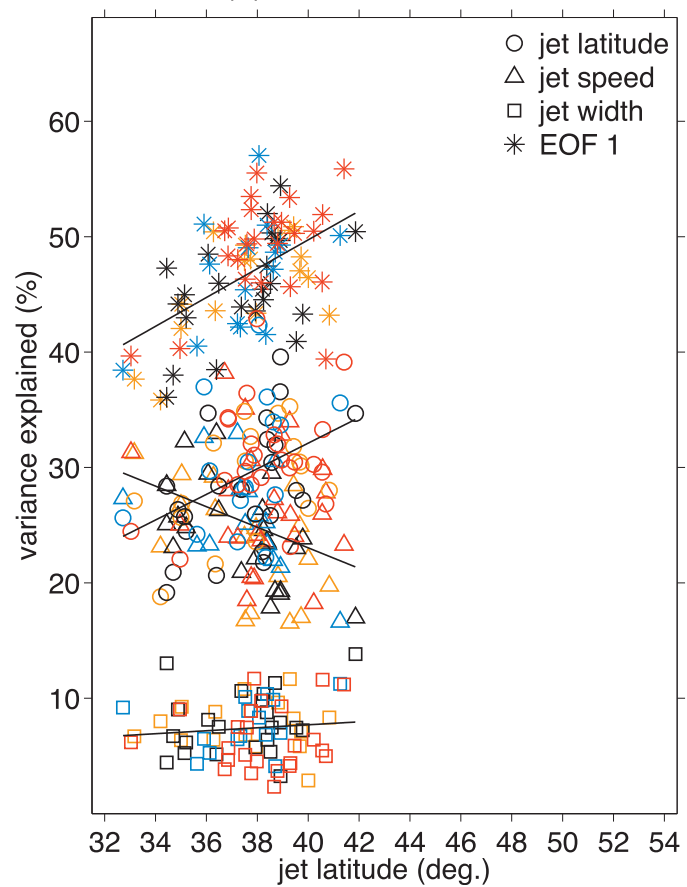

(b) North Pacific, JJA

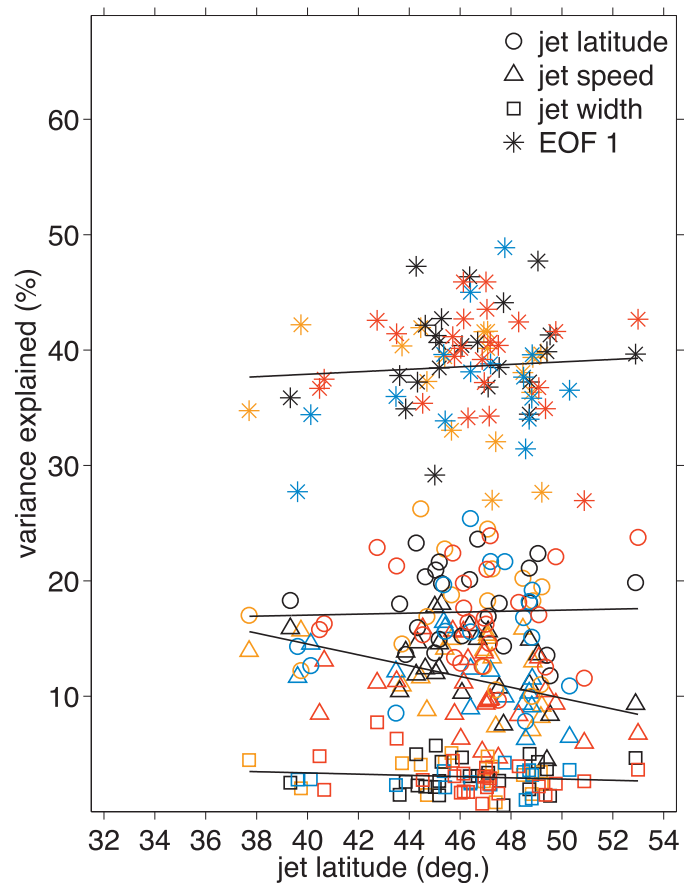

FIG. 13. As in Fig. 6, but for the North Pacific sector during (a) winter (DJF) and (b) summer (JJA). 
TABLE 3. Multimodel mean jet latitudes, poleward jet shift, and response of the jet variability between the Historical and RCP8.5 experiments. Note that the average shift is not necessarily the difference between the Historical and RCP 8.5 mean jet positions since the shift is calculated only from models with data for both the Historical and RCP8.5 experiments, while the average jet positions are calculated with all data available.

\begin{tabular}{lcccc}
\hline \hline \multicolumn{1}{c}{ Region } & $\begin{array}{c}\text { Historical }(1980-2004) \\
\text { jet lat }\left({ }^{\circ} \mathrm{N}\right)\end{array}$ & $\begin{array}{c}\text { RCP8.5 }(2076-99) \\
\text { jet lat }\left({ }^{\circ} \mathrm{N}\right)\end{array}$ & $\begin{array}{c}\text { Lat shift } \\
\left({ }^{\circ} \text { poleward }\right)\end{array}$ & $\begin{array}{c}\text { Variability } \\
\text { response }\end{array}$ \\
\hline Southern Hemisphere & -47.8 & -49.5 & 1.8 & $\begin{array}{c}\text { Less wobbling, more pulsing } \\
\text { North Atlantic }\end{array}$ \\
North Pacific & 46.9 & 48.1 & 1.1 & $\begin{array}{l}\text { Less wobbling, more pulsing } \\
\text { More wobbling, less pulsing }\end{array}$ \\
\hline
\end{tabular}

equatorward in the winter than the summer, and the dependence of the variability on the mean jet position in DJF is similar to that of the annual mean (Fig. 6c), when the subtropical jet is strong. In the summer, however, the subtropical jet is weak (not shown), and the eddy-driven jet is farther poleward. In this instance, we find no significant dependence of $\tilde{Z}_{\text {lat }}$ on the mean jet position although $\tilde{Z}_{\text {spd }}$ does still exhibit a negative correlation with jet latitude. These seasonal plots support the dynamical mechanism linking decreased jet wobbling to the presence of a strong subtropical jet, but more work is needed to clarify the role of the seasonality of subtropical winds in modulating the eddy-driven jet's variability response.

We find that there is also a strong seasonal subtropical jet in the Southern Hemisphere Pacific Ocean during winter, which can influence the eddy-driven jet variability there (Barnes and Hartmann 2010a). Again, we have no room to discuss the details here, but in future work we will investigate the seasonality of the Southern Hemisphere eddy-driven jet response in a zonally asymmetric framework.

\section{Conclusions}

We have demonstrated a robust response of the eddydriven jets to climate change in the CMIP5 multimodel mean ensemble and, more importantly, have highlighted the response of their variability to increased greenhouse gas forcing. Our main findings are summarized in Table 3. In all three basins, models predict a $1^{\circ}-2^{\circ}$ poleward shift of the jets by the end of the twenty-first century. With this shift, we find that jet variability in the North Atlantic and Southern Hemisphere becomes less of a meridional jet wobble and more of a change in jet speed, while jet variability in the North Pacific becomes more of a wobble and less of a pulse. We argue that these different responses can be dynamically linked, through Rossby wave breaking, to the mean latitude of the jet relative to the subtropics and poles.

These results highlight that the leading pattern of variability for a given model experiment can be a strong function of the mean state, specifically of the mean jet latitude. As a consequence, using the variability patterns (e.g., as defined by EOFs) from one model integration to quantify variability in another integration or in another model can lead to severe errors. Our findings suggest that GCM biases in the mean jet position will directly relate to biases in the GCM's atmospheric variability, given the dependence of the variability pattern on jet latitude. Since most GCMs have large equatorward jet biases in the North Atlantic and Southern Hemisphere, our results suggest that GCMs will also exhibit strong biases in their tropospheric variability. Finally, since some models project very large responses in the jet position (i.e., shifts of up to $5^{\circ}$ poleward), it is possible that the leading pattern of variability in the future may be quite different from the familiar north-south wobble of the jet.

Eddy-driven jet variability is strongly tied to the position of the storm tracks (Limpasuvan and Hartmann 1999), regional weather (Hurrell et al. 2003), blocking anticyclone frequency (Woollings et al. 2008), and wind driving of the oceans with implications for sea ice extent (Hall and Visbeck 2002; Holland and Kwok 2012). With such diverse ties to physical processes within the troposphere and at Earth's surface, changes in the dominant forms of variability of the eddy-driven jets are likely to have important global impacts.

Acknowledgments. We thank Nicholas W. Barnes and Haibo Liu for obtaining the CMIP5 data. We acknowledge the Global Modeling and Assimilation Office (GMAO) and the Goddard Earth Sciences Data and Information Services Center (GES DISC) for the dissemination of MERRA. We acknowledge the World Climate Research Programme's Working Group on Coupled Modelling, which is responsible for CMIP, and we thank the climate modeling groups (listed in Table 1 of this paper) for producing and making available their model output. For CMIP the U.S. Department of Energy's Program for Climate Model Diagnosis and Intercomparison provides coordinating support and led development of software infrastructure in partnership with the Global Organization for Earth System Science Portals. EAB is funded by a NOAA Climate 
(a) Southern Hemisphere

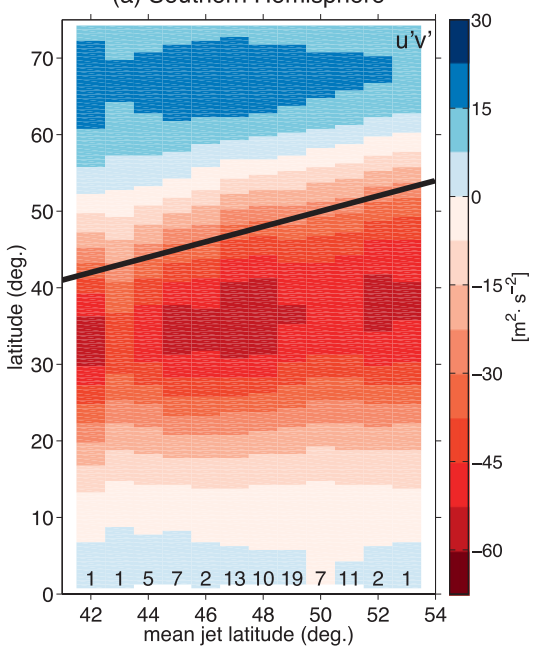

(b) North Atlantic

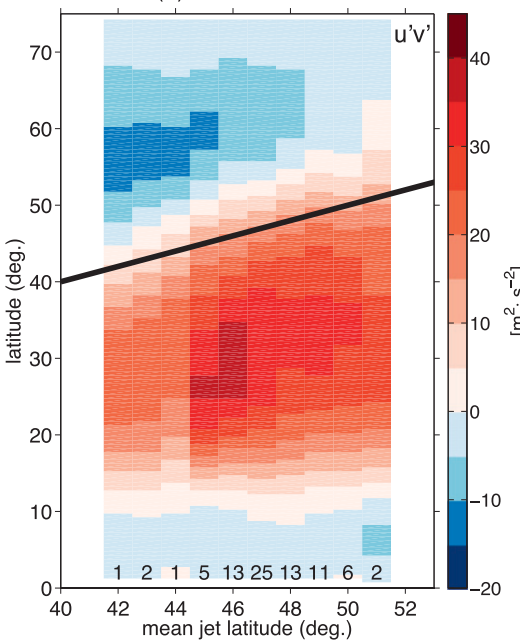

(c) North Pacific

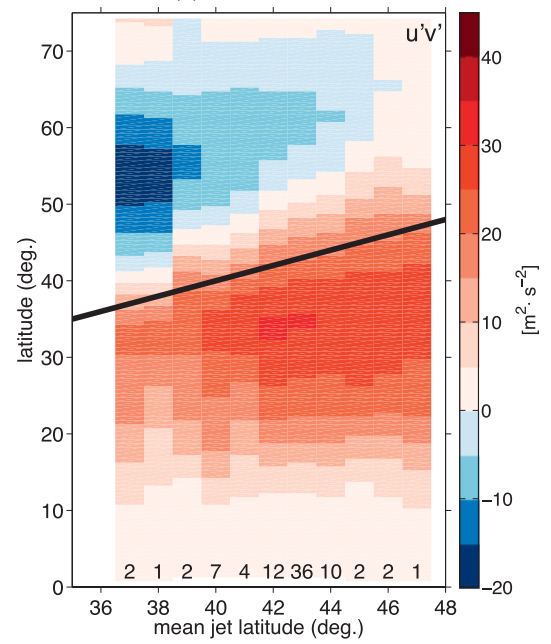

FIG. B1. (a)-(c) Zonally integrated, 250-hPa eddy-momentum flux profiles vs mean jet latitude for the three sectors, respectively, where the eddies are defined with a 10-day high-pass filter. Note that the colors in (a) have been flipped for easier comparison with (b) and (c). Model results have been grouped into $1^{\circ}$ jet latitude bins and the profiles averaged together and the number of profiles in each jet latitude bin is denoted along the $x$ axis. The thick line denotes the 1:1 line and thus the position of the mean jet.

and Global Change Fellowship through the University Corporation of Atmospheric Research Visiting Science Program. LMP is funded, in part, by a grant of the U.S. National Science Foundation to Columbia University.

\section{APPENDIX A}

\section{Method to Calculate Explained Variance}

The area-weighted percent variance explained of the sector-average anomalous zonal wind $[\tilde{u}=\tilde{u}(t, j)]$ by the anomalous time series $\tilde{X}=\tilde{X}(t, j)$, where $j$ is an index over latitude and $t$ is an index over time, is defined as

$$
\frac{\sum_{j} \operatorname{Corr}\left(\tilde{u}_{j}, \tilde{X}_{j}\right)^{2} \times \operatorname{Var}\left(\tilde{u}_{j}\right) \times \cos _{j}}{\sum_{j} \operatorname{Var}\left(\tilde{u}_{j}\right) \times \cos _{j}} \times 100 .
$$

Here $\cos _{j}$ denotes the cosine of the $j$ th latitude and the sum is performed between $25^{\circ}$ and $90^{\circ}$ latitude for each sector. The operator Corr denotes the correlation defined as

$$
\operatorname{Corr}(a, b)=\frac{\sum_{t}[a(t)-\bar{a}][b(t)-\bar{b}]}{\sqrt{\sum_{t}[a(t)-\bar{a}]^{2} \sum_{t}[b(t)-\bar{b}]^{2}}}
$$

for two time series $a(t)$ and $b(t)$ with means $\bar{a}$ and $\bar{b}$, respectively. The operator Var denotes the variance defined as

$$
\operatorname{Var}(a)=\frac{1}{N} \sum_{t}[a(t)-\bar{a}]^{2}
$$

where $N$ is the length of $a$.

In calculating the percent variance explained, days were removed from the calculation when any of the three time series $\left(\tilde{Z}_{\text {lat }}, \tilde{Z}_{\text {spd }}\right.$, and $\left.\tilde{Z}_{\text {wdt }}\right)$ was undefined. The quantity $\tilde{Z}_{\text {wdt }}$ was the most often undefined (jet profile never reached half of its maximum), and this typically occurred less than $2 \%$ of the time. We have confirmed that this step does not qualitatively affect the final conclusions.

\section{APPENDIX B}

\section{Eddy-Momentum Flux Profiles}

Figure B1 is similar to Fig. 10, except here we plot the zonal-mean $250-\mathrm{hPa}$ eddy-momentum flux profiles, where eddies are calculated using a 10-day high-pass Lanczos filter with 41 weights (see Duchon 1979 for details). The eddy-momentum flux is another metric to quantify wave propagation and we have included it here since eddy-momentum flux divergence occurs where Rossby waves break and it is easier to diagnose this divergence than track individual Rossby waves. Similar to Figs. 10a,b, the eddy-momentum flux at high latitudes decreases for jets found at higher latitudes, while the momentum flux equatorward of the jet axis follows the jet. In the Southern Hemisphere especially, the equatorward (positive values, blue shading) momentum fluxes 
on the poleward jet flank do not move with the jet axis, as is the case for the cyclonic Rossby wavebreaking frequencies. In the North Pacific, the polewardmomentum fluxes are larger than the other two basins, consistent with the wave-breaking occurrence (Fig. 10h); however, the poleward-momentum fluxes also appear to decrease somewhat as the jet is located at higher latitudes. This is in agreement with the Rossby wave-breaking analysis.

\section{REFERENCES}

Baldwin, M. P., and T. J. Dunkerton, 1999: Propagation of the Arctic Oscillation from the stratosphere to the troposphere. J. Geophys. Res., 104, 30 937-30 946, doi:10.1029/1999JD900445.

Barnes, E. A., and D. L. Hartmann, 2010a: Dynamical feedbacks of the southern annular mode in winter and summer. J. Atmos. Sci., 67, 2320-2330.

— and - 2010b: Influence of eddy-driven jet latitude on North Atlantic jet persistence and blocking frequency in CMIP3 integrations. Geophys. Res. Lett., 37, L23802, doi:10.1029/ 2010GL045700.

— and - 2010c: Testing a theory for the effect of latitude on the persistence of eddy driven jets using the CMIP3 simulations. Geophys. Res. Lett., 37, L15801, doi:10.1029/2010GL044144.

— , and — 2011: Rossby-wave scales, propagation, and the variability of eddy-driven jets. J. Atmos. Sci., 68, 2893-2908.

— and - 2012: Detection of Rossby wave breaking and its response to shifts of the midlatitude jet with climate change. J. Geophys. Res., 117, D09117, doi:10.1029/2012JD01746.

Deser, C., 2007: On the teleconnectivity of the "Arctic Oscillation." J. Climate, 20, 4751-4767.

Duchon, C. E., 1979: Lancos filtering in one and two dimensions. J. Appl. Meteor., 18, 1016-1022.

Eichelberger, S. J., and D. L. Hartmann, 2007: Zonal jet structure and the leading mode of variability. J. Climate, 20, 5149-5163.

Garfinkel, C. I., D. W. Waugh, and E. P. Gerber, 2013: The effect of tropospheric jet latitude on coupling between the stratospheric polar vortex and the troposphere. J. Atmos. Sci., 26, 2077-2095.

Gerber, E. P., L. M. Polvani, and D. Ancukiewicz, 2008: Annular mode time scales in the Intergovernmental Panel on Climate Change Fourth Assessment Report models. Geophys. Res. Lett., 35, L22707, doi:10.1029/2008GL035712.

_- and Coauthors, 2010: Stratosphere-troposphere coupling and annular mode variability in chemistry-climate models. J. Geophys. Res., 115, D00M06, doi:10.1029/2009JD013770.

Hall, A., and M. Visbeck, 2002: Synchronous variability in the Southern Hemisphere atmosphere, sea ice, and ocean resulting from the annular mode. J. Climate, 15, 3043-3057.

Hartmann, D. L., 2007: The atmospheric general circulation and its variability. J. Meteor. Soc. Japan, 85B, 123-143.

- , and F. Lo, 1998: Wave-driven zonal flow vacillation in the Southern Hemisphere. J. Atmos. Sci., 55, 1303-1315.

Holland, P. R., and R. Kwok, 2012: Wind-driven trends in Antarctic sea-ice drift. Nat. Geosci., 5, 872-875, doi:10.1038/NGEO1627.

Hurrell, J. W., Y. Kushnir, G. Ottersen, and M. Visbeck, 2003: An overview of the North Atlantic Oscillation. The North Atlantic Oscillation: Climatic Significance and Environmental Impact, Geophys. Monogr., Vol. 134, Amer. Geophys. Union, 1-35.

Kidston, J., and E. Gerber, 2010: Intermodel variability of the poleward shift of the austral jet stream in the CMIP3 integrations linked to biases in the 20th century climatology. Geophys. Res. Lett., 37, L09708, doi:10.1029/2010GL042873.

Limpasuvan, V., and D. L. Hartmann, 1999: Eddies and the annular modes of climate variability. Geophys. Res. Lett., 26, 31333136.

Lorenz, D. J., and D. L. Hartmann, 2001: Eddy-zonal flow feedback in the Southern Hemisphere. J. Atmos. Sci., 58, 3312-3327. , and - 2003: Eddy-zonal flow feedback in the Northern Hemisphere winter. J. Climate, 16, 1212-1227.

Martius, O., C. Schwierz, and H. Davies, 2007: Breaking waves at the tropopause in the wintertime Northern Hemisphere: Climatological analyses of the orientation and the theoretical LC1/2 classification. J. Atmos. Sci., 64, 2576-2592.

Meehl, G. A., C. Covey, T. Delworth, M. Latif, B. McAvaney, J. F. B. Mitchell, R. J. Stouffer, and K. E. Taylor, 2007: The WRCP CMIP3 multimodel dataset: A new era in climate change research. Bull. Amer. Meteor. Soc., 88, 1383-1394.

Miller, R. L., G. A. Schmidt, and D. T. Shindell, 2006: Forced annular variations in the 20th century Intergovernmental Panel on Climate Change Fourth Assessment Report models. J. Geophys. Res., 111, D18101, doi:10.1029/2005JD006323.

Rienecker, M., and Coauthors, 2011: MERRA: NASAs ModernEra Retrospective Analysis for Research and Applications. J. Climate, 24, 3624-3648.

Stephenson, D. B., V. Pavan, M. Collins, M. M. Junge, and R. Quadrelli, 2006: North Atlantic Oscillation response to transient greenhouse gas forcing and the impact on European winter climate: A CMIP2 multi-model assessment. Climate Dyn., 27, 401-420, doi:10.1007/s00382-006-0140-x.

Strong, C., and G. Magnusdottir, 2008: Tropospheric Rossby wave breaking and the NAO/NAM. J. Atmos. Sci., 65, 2861-2876.

Swart, N., and J. Fyfe, 2012: Observed and simulated changes in the Southern Hemisphere surface westerly wind-stress. Geophys. Res. Lett., 39, L16711, doi:10.1029/2012GL052810.

Thompson, D. W., and J. M. Wallace, 2000: Annular modes in the extratropical circulation. Part I: Month-to-month variability. J. Climate, 13, 1000-1016.

Vallis, G. K., E. P. Gerber, P. J. Kushner, and B. A. Cash, 2004: A mechanism and simple dynamical model of the North Atlantic Oscillation and annular modes. J. Atmos. Sci., 61, 264-280.

Visbeck, M., 2009: A station-based southern annular mode index from 1884 to 2005. J. Climate, 22, 940-950.

Walker, G. T., 1924: Correlation in seasonal variation of weather, IX. A further study of world weather. Mem. Indian Meteor. Dep., 24, 275-333.

Wernli, H., and M. Sprenger, 2007: Identification and ERA-15 climatology of potential vorticity streamers and cutoffs near the extratropical tropopause. J. Atmos. Sci., 64, 1569-1580.

Wittman, M. A., A. J. Charlton, and L. M. Polvani, 2005: On the meridional structure of annular modes. J. Climate, 18, 21192122 .

Woollings, T., and M. Blackburn, 2012: The North Atlantic jet stream under climate change and its relation to the NAO and EA patterns. J. Climate, 25, 886-902.

_ B. Hoskins, M. Blackburn, and P. Berrisford, 2008: A new Rossby wave-breaking interpretation of the North Atlantic Oscillation. J. Atmos. Sci., 65, 609-626.

_ A. Hannachi, and B. Hoskins, 2010: Variability of the North Atlantic eddy-driven jet stream. Quart. J. Roy. Meteor. Soc., 136, 856-868, doi:10.1002/qj.625.

Yin, J. H., 2005: A consistent poleward shift of the storm tracks in simulations of 21st century climate. Geophys. Res. Lett., 32, L18701, doi:10.1029/2005GL023684 